\title{
REVIEW
}

\section{Lipid-nanostructure hybrids and their applications in nanobiotechnology}

\author{
Young Kwang Lee ${ }^{1}$, Hyojin Lee ${ }^{1}$ and Jwa-Min Nam
}

Cell membranes contain a variety of lipids and functional proteins that offer active platforms that organize the membrane components into functional assemblies and perform biologically important reactions. The dynamic and complex nature of the membranes makes them attractive materials, but at the same time, researchers report substantial experimental uncertainty in controlling the membrane reactions and extracting valuable information. The fascinating new structures and properties of nanomaterials could be utilized in addressing aforementioned issues, and the design and synthesis of lipid-nanostructure hybrids could be beneficial to the research areas in lipid-membrane biotechnology and nanobiotechnology. These hybrid structures possess dimensions that are comparable to that of biological molecules and structures and physicochemical properties that arise from both lipids and nanomaterials. Therefore, lipid-nanostructure hybrids offer additional options for control of the synthesized structures, provide new insight in understanding nanostructures and biological systems and allow the mimicking of functional subcellular membrane components and monitoring of the membrane-associated reactions in a highly sensitive and controllable manner. In this review, we present recent advances in the synthesis of various lipid-nanostructure hybrids and the application of these structures in biotechnology and nanotechnology. We further describe the scientific and practical applications of lipid-nanostructure hybrids for detecting membrane-targeting molecules, interfacing nanostructures with live cells and creating membrane-mimicking platforms to investigate various intercellular processes.

NPG Asia Materials (2013) 5, e48; doi:10.1038/am.2013.13; published online 17 May 2013

Keywords: biosensor and nano-bio interface; cell membrane; nanopattern; nanopore; nanostructure; nanotopography; supported lipid bilayer

\section{INTRODUCTION}

Cell membranes are primarily composed of a variety of lipids in the form of two asymmetric leaflets and functional proteins. The membranes define a cellular boundary and provide a basic platform for tight regulation of many biological processes, including material transport, signal transduction, trafficking, pathogenic pathways, intercellular organization and response to the extracellular matrix. These processes are primarily involved in the conformational change and rearrangement of membrane constituents, which accelerate the temporal and spatial heterogeneity in cell membranes. Such a dynamic and complex nature means that the native cell membranes are sources of experimental uncertainty, and great demands exist for the ability to precisely assess the physical and chemical specificity of cell membranes for the reduction of experimental complexity and uncertainty and the ability to leverage the key features of lipids, including lateral fluidity, structural versatility and compatibility with other cell membrane components. Phospholipid vesicles, which were first observed in 1965 by Bangham et al., ${ }^{1}$ are versatile supramolecular assemblies that can be readily prepared with diverse shapes, sizes and compositions. The physical properties of lipid vesicles are similar to those of native cell membranes in which the cellular interior is isolated from the external environment by lipid and protein-rich dynamic boundaries. The capacities of lipid vesicles for tuning chemical composition and embedding and encapsulating various materials have facilitated the use of the vesicles in many fields, including cell membrane-mimicking materials and drug delivery. Subsequently, supported lipid bilayers (SLBs) or self-assembled lipid membranes on solid substrates were developed by Tamm and McConnell. ${ }^{2,3}$ The SLB is a model membrane platform that provides robust artificial cell membranes in a synthetically controllable manner, recapitulates key membrane functions and allows the study of structural changes and reactions in membranes on a surface through various optical and characterization tools. During the past two decades, SLBs have been decorated with a variety of membrane-associated molecules, coated on various solid supports and coupled with micro-fluidic and microarray techniques. ${ }^{4,5}$ These advances have enabled the development of SLB-based membrane-mimicking systems for sensing membrane reactions, studying intercellular signaling and separating membrane species. $^{6-9}$ 
To simulate and monitor various cell membrane-based processes, the lateral heterogeneity in a subcellular dimension should be reproducibly created and the molecular interaction should be read out with high reproducibility and high sensitivity. Nanomaterials have generated great interest because their size, shape, dimensions and composition-dependent properties are comparable to those of biological molecules and structures, allowing the investigation of biological phenomena at a subcellular level. Recent advances in the synthesis and fabrication techniques for targeted nanostructures (that is, metallic nanoparticles, semiconductor nanoparticles, metal oxide nanoparticles, nanotubes/wires, nanopores and nanotopographic surfaces with high controllability) have boosted the development of new platforms and methodologies designed to extract valuable information from complex biological processes. Such breakthroughs have taken advantage of the unique physicochemical consequences (for example, surface plasmon resonance, superparamagenetism, excellent luminescence properties, high surface-to-volume ratio and high signal-tonoise ratio, and so on) that arise from varying the size, composition, shape and arrangement of nanomaterials. ${ }^{10-12}$ Plain lipid structures can be transformed into versatile hybrid nanostructures if nanostructures are used as a solid support. Lipid-nanostructure hybrids often exhibit new structures and properties that cannot be obtained with single components. The synthesis of lipidnanostructure hybrids also allows the construction of artificial cell membrane environments on the surface of nanomaterials that can adopt biomolecules with little perturbation of biological activity, molecular structure and orientation. This option creates a surface containing both physical and chemical cues at the nanoscale to which can cells respond, paving the way for the development of livingnonliving interfacing platforms. In addition, the hybrid system can be used in a wide range of applications, from nanomaterial-based biosensors to the detection of molecular reactions occurring on the membrane surface. The lipid bilayers can be also useful for developing new working principles based on unique properties, such as high electrical resistance and lateral fluidity. Thus, hybrids of lipid bilayers and nanomaterials provide exciting new opportunities for cell membrane studies that are unprecedented in terms of sensitivity, spatial resolution and controllability. However, the synthetic methods and the consequences of forming such hybrid structures have not yet been fully studied or understood, and solutions to these issues are critical for the reliable and practical use of nanostructures and lipids in both materials and biological applications. In this work, we review the recent advances in the design and synthesis of SLB-based lipidnanostructure hybrids and the use of these structures in applications of biotechnology and nanotechnology. In this review, in addition to the typical synthetic schemes for liposome-nanostructure hybrids and applications in analytical applications, ${ }^{13,14}$ we focus primarily on the applications of lipid-nanostructure hybrids for detecting biomolecules that interact with cognate membrane receptors and creating live cellmimicking and interfacing platforms for the investigation of intercellular processes that take place at the membrane junctions.

\section{FORMATION OF LIPID-NANOSTRUCTURE HYBRIDS AND THEIR STRUCTURES AND PROPERTIES}

If lipid films are mechanically supported by nanostructured substrates, the resulting hybrid structure is largely determined by the structure and properties of the supporting nanomaterials because the morphology of the lipid layers duplicate that of the underlying supports while also maintaining close surface proximity. In this case, the lipid molecules should be adsorbed and subsequently rearranged via self-assembly on the nanostructured surface. However, there is rising concern with respect to preserving the biological functions of lipid membranes and proteins when they are integrated with nanostructured supports because the nanotopographic features (characterized by rough surface, high curvature and/or altered dimensionality) can have profound effects on the structures and properties of the resulting lipid membranes and their components in terms of formation kinetics, stability, gel-fluid coexistence regime, and the dynamics of lipid molecules. Therefore, understanding such interactions between the membrane and the underlying nanostructured substrate and selecting a suitable synthetic method will allow the rational design and synthesis of nano-bio hybrids with high activity.

\section{Lipid-bilayer-modified nanotopographic substrates}

Conventional planar SLBs are commonly formed using the vesicle fusion method. When small unilamellar vesicles, typically with a diameter of $\sim 100 \mathrm{~nm}$, are incubated with hydrophilic solid supports, the vesicles are adsorbed on the surface and deformed by interaction with the solid substrate. ${ }^{15}$ The support-induced stress is further enhanced by adsorption of the vesicles in their vicinity. The adsorbed liposomes rupture and form a continuous lipid bilayer on the solid support at a certain surface density of vesicles that corresponds to a critical vesicular coverage. This vesicle fusion method has also been widely used for the formation of lipidnanostructure hybrids. However, the formation kinetics and membrane physics of lipid-nanostructure hybrids are significantly different from those of planar SLBs.

Notably, recent experimental results have shown that a certain degree of surface curvature induces the loss of structural integrity as well as pore formation of lipid membranes on nanostructured substrates. ${ }^{16,17}$ An atomic force microscopy study revealed that substrate-supported silica nanoparticles with diameters ranging from 1.2 to $22 \mathrm{~nm}$ pierced the $\mathrm{L}-\alpha$-dimyristoyl phosphatidylcholine bilayer, whereas particles outside of that size range supported the $\mathrm{L}-\alpha$ dimyristoyl phosphatidylcholine membrane without pore formation (Figure 1a). The incomplete bilayer formation was attributed to the unfavorable bending energy of the membrane forming a highly curved structure. A cryo-electron microscopy study revealed that the detailed rupture mechanism of small unilamellar vesicles that formed a complete bilayer on highly curved silica nanoparticles is involved with individual rupture processes below a local critical density of vesicles required for promoting the formation of the SLB on a planar silica surface (Figure $1 \mathrm{~b}$ ).$^{18}$ This study also showed the importance of the lipid composition in constructing a continuous and intact lipid bilayer. Complete SLB formation on a silica nanoparticle was achieved using liposomes with positive, neutral or low net negative charges, whereas liposomes with a high net negative charge remained unruptured on silica nanoparticles (Figure 1b). The size of the small unilamellar vesicles also has a profound effect on the kinetics of the vesicle-to-bilayer transformation on nanostructures pitted with nanoholes. ${ }^{19}$ Studies aimed at understanding the SLB formation on nanotopographic surfaces and designing localized surface plasmon resonance (LSPR)-based membrane biosensors consisting of lipid-covered metal nanoparticles or nanoholes have also been reported. ${ }^{20,21}$

\section{Lipid bilayers on nanopatterns}

Lipid bilayers are selectively assembled as fluids on the surface of specific materials, typically silica. A structure that hampers the formation of a bilayer or the sliding of lipid molecules on the surface can be used as a diffusion barrier. The Boxer group has proposed and 
a
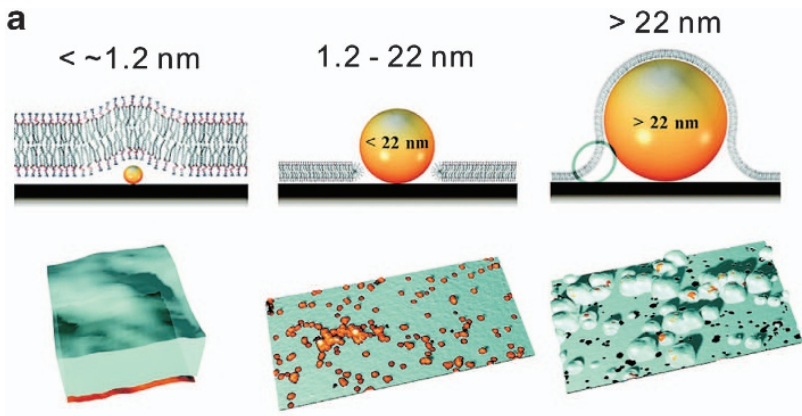

b
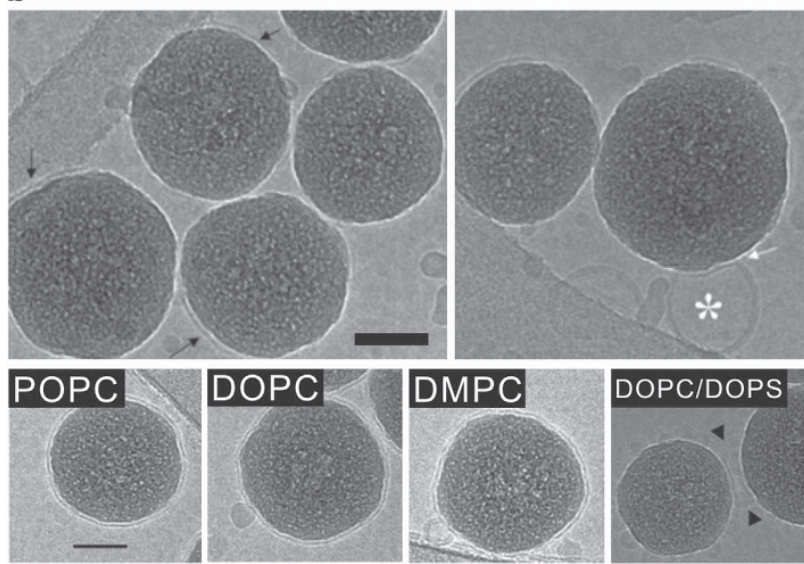

Figure 1 Supported lipid bilayers (SLBs) on nanotopographic solid substrates. (a) Top: schemes illustrate the structures of lipid bilayers in the presence of silica nanoparticles with varying size. Bottom: corresponding atomic force microscopy (AFM) images of upper schemes. ${ }^{16}$ (b) Top: cryotransmission electron microscope images from mixtures of silica particles and 1,2-dioleoyl-sn-glycero-3-phosphocholine (DOPC)/1,2-dioleoyl-sn-glycero3-phospho-L-serine (DOPS) (4:1) small unilamellar vesicles (SUVs), quickly frozen after a 1-min incubation time. Most particles present one or two patches of lipid bilayer at their interfaces (black arrows). Few particles present the adsorbed, unruptured vesicles at their surface (white asterisks). Bottom: SLBs formed with SUVs containing neutral lipids only (DOPC, 1-palmitoyl-2-oleoyl-sn-glycero-3-phosphocholine (POPC) and L- $\alpha$-dimyristoyl phosphatidylcholine (DMPC)) and a high concentration of negatively charged lipids (DOPC/DOPS, 1:1). The scale bars are $50 \mathrm{~nm} .{ }^{18}$

extensively studied methodologies for fabricating patterned lipid bilayers via various patterning techniques. ${ }^{22,23}$ These techniques have been used to fabricate multi-compositional lipid microarrays and to control membrane dynamics. ${ }^{24}$ The incubation of small unilamellar vesicles with a barrier-patterned substrate leads to the formation of spatial patterns of lipid membranes that act to confine molecular motions. ${ }^{7,25,26}$ Advances in patterning techniques with various materials and spatial resolutions provide opportunities for fabricating nanopatterned fluid surfaces that modulate the motion of membrane species in the nanometer dimension.

Electron-beam lithography has been commonly used to fabricate Cr nanopatterns that completely disrupt the lipid bilayer, and thus, they could serve as passive barriers that hinder the lateral diffusion of lipids (Figure 2a). ${ }^{27}$ The lift-off process resist patterns generated with electron-beam lithography typically produce $\mathrm{Cr}$ barriers a few nanometers high and a hundred nanometers wide. These barriers are used to engineer the spatial organization of cell membrane receptors for interfacing with living cells. However, this method may be useful for generating patterns in a relatively small area (several micrometers in size), and the exposure of the substrate to a highvacuum environment limits the use of this method for the direct and multi-component patterning of biomolecules.

Dip-pen nanolithography (DPN) is a scanning probe-based lithographic technique that directly delivers ink molecules from the tip of an atomic force microscope to an underlying substrate. ${ }^{28} \mathrm{~A}$ massively parallel DPN technique has been introduced to fabricate multiplexed patterns of various molecules and high-throughput molecular prints. ${ }^{29}$ In the patterning of lipid molecules, poly(diallydimethylammonium chloride) was used as the inking molecule in DPN to introduce diffusion barriers and bio-functionalization sites in the SLB. ${ }^{30}$ After SLB formation, the resulting poly(diallydimethylammonium chloride) patterns provided a template for protein adsorption and the inhibition of lipid diffusion. In certain cases, disjointed poly(diallydimethylammonium chloride) lines were formed, and such structures could be used to mimic the restricted diffusion in cells and to study the nonBrownian diffusion behavior of membrane-bound molecules. Phospholipids, the major constituents of cell membranes, were directly transferred and formed in the patterned fluid lipid films using DPN. ${ }^{31}$ Under the appropriate humidity and scan speed, the thickness of a deposited lipid layer can be controlled to a single bilayer thickness, and the lateral resolution can be reduced to sub-100-nm levels. The massively parallel and direct delivery with DPN of multi-component lipids and lipophilic materials to a solid surface on multiple length scales enables the mimicking of heterogeneous micro- and nanodomains of native cell membranes that are similar to lipid rafts (Figure $2 \mathrm{~b}$ ). ${ }^{32}$ An atomic force microscopy tip can locally remove a preexisting thin film from a solid support; this method is known as nanoshaving lithography (Figure 2c). ${ }^{33}$ When a shaved region is backfilled with another lipid layer, a lipid pattern as narrow as $50 \mathrm{~nm}$ can be formed. In another case, a nanoarray of protein modification sites in SLBs was introduced via block copolymer micelle nanolithography (Figure 2d). ${ }^{34}$ The self-assembly of gold ion-containing diblock copolymer micelles into a hexagonal array and the subsequent air plasma treatment produced $\sim 5-7-\mathrm{nm}$ gold nanoparticles on the substrate, and the SLB was formed on this substrate using the vesicle fusion method. This approach allows for control of the spacing between particles in an array via tuning of the polymer molecular weight and does not require complex patterning procedures. Gold nanoparticles embedded in the SLB were modified with various biological molecules via thiol linkers. This approach offers methods for functionalizing proteins or other ligands to the fluid lipid component, the fixed nanoparticles or both lipids and nanoparticles over a large area.

\section{Lipid-1D-nanostructure hybrids}

The unique geometric features of one-dimensional (1D) nanostructures allow them to be efficiently integrated into functional devices in a high-density array format, and their nanoscale-size effects (such high sensitivity to surface-binding events) can be leveraged for various sensing applications. ${ }^{35}$ The introduction of a lipid bilayer to a 1D nanostructured surface allows the integration of various functions and structures of membrane-associated biomolecules on the arrayed 1D nanostructures and the imitation of the chemical and mechanical properties of cell membrane-related fibrous structures on engineered 1D nanostructures.

The supramolecular self-assembly of lipids on carbon nanotubes (CNTs) above the critical micellar concentration was observed via transmission electron microscopy. ${ }^{36}$ In this approach, lipids were adsorbed on the CNT surface in the shape of a regular ring, helix or double helix of a half cylinder, depending on the symmetry and diameter of CNTs, and these results strongly support the hypothesis 
a

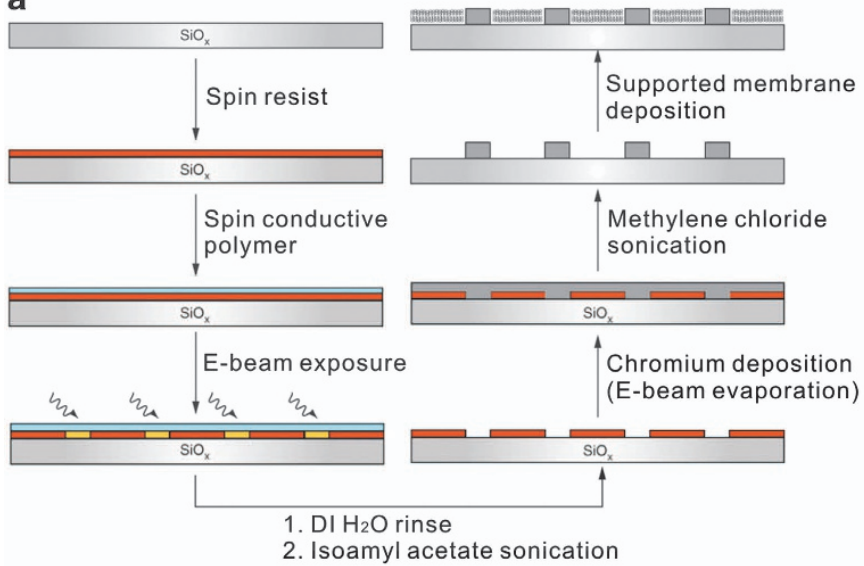

b
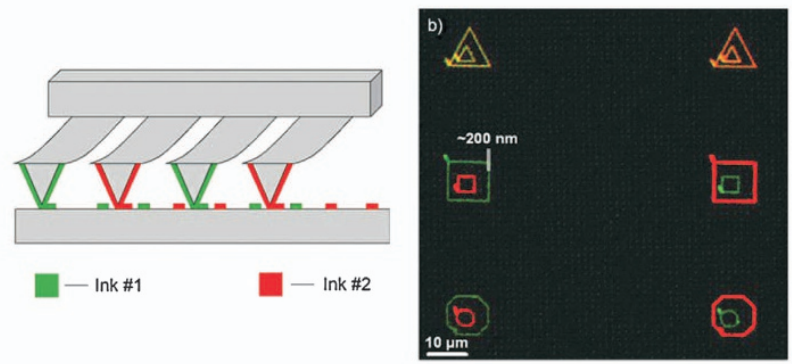

d

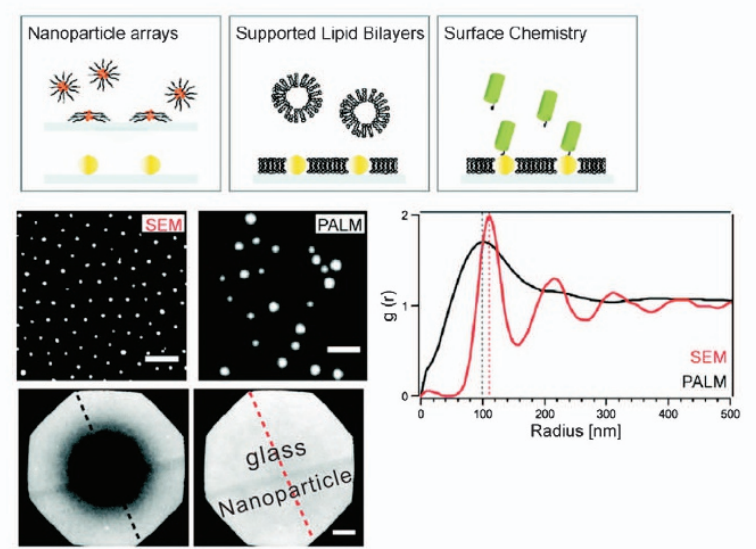

Figure 2 Formation of lipid-nanopatterned hybrids. (a) Fabrication of metal lines using electron-beam lithography and the subsequent formation of patterned lipid bilayers. ${ }^{27}$ (b) Left: schematic illustration of parallel patterning of different lipids on subcellular scales with a multiplexing dip-pen nanolithography (DPN) cantilever array. Right: fluorescence images of multilayered 1,2-dioleoyl-sn-glycero-3-phosphocholine (DOPC) structures containing fluorophore-labeled lipids (rhodamine/red and fluorescein/green). ${ }^{32}$ (c) Left: schematic illustration of atomic force microscopy (AFM)-based nanoshaving lithography for nanoscale supported lipid bilayer (SLB) formation. Right: epifluorescence images of a nanoshaved bovine serum albumin (BSA) monolayer (top) and SLB lines (bottom). The scale bar is $3 \mu \mathrm{m} .{ }^{33}$ (d) Top: schematic overview of nanoparticle array-embedded SLBs. Middle: from the left, reference SEM image of gold nanoparticle arrays and photoactivated localization microscopy (PALM) image of individual ephrin-mEos-His10 molecules conjugated on the same gold nanoparticle array. The scale bar is $200 \mathrm{~nm}$ in both cases. The right figure represents comparison of the radial distribution function from the full PALM image (black) and the reference SEM image (red). Bottom: fluorescence recovery after photobleaching (FRAP) on a bilayer of DOPC doped with 0.5\% Texas Red-1,2-dihexadecanoyl-sn-glycero-3-phosphoethanolamine (DHPE). ${ }^{34}$

that lipids can be organized along the graphite network. Noncovalent surface decoration with amphiphiles is a promising approach to preserve the inherent properties of CNTs and to render CNTs soluble in aqueous environments. Although this static lipid-CNT hybrid is sufficiently robust and offers many advantages for surface engineering of CNTs, the formation of a fluid lipid bilayer on the CNT and the preservation of the nature-like structures and functions of the membrane-associated biomolecules are highly challenging tasks. The self-assembly of lipid molecules into a fluidic 1D nanostructure on a CNT template was reported, and the lipid lateral fluidity was tested by tuning the diameter of the CNTs (Figure 3a). ${ }^{37}$ For support of the hydrophilic head groups of the lipid bilayer, the CNTs were wrapped with polyelectrolytes in a layer-by-layer manner. The assembled polyelectrolytes electrostatically stabilized the lipid bilayer in a $1 \mathrm{D}$ configuration and allowed control of the diameter of the structure. The CNT-templated 1D lipid bilayer displayed structural continuity and retained the lateral mobility of lipid molecules, even for highly curved structures. However, their diffusion coefficients were nearly two orders of magnitude smaller than that of other polymersupported planar lipid bilayers. Later, 1D nanostructure-supported lipid membrane hybrid structures with high diffusion coefficients and nearly $100 \%$ lipid mobility were reported (Figure $3 \mathrm{~b}$ ). ${ }^{38}$ In this work, amorphous silicon-coated CNTs with diameters ranging from 20 to $200 \mathrm{~nm}$ were suspended across micro-fabricated channels in $\mathrm{Si} / \mathrm{SiO}_{2}$ substrates that were used as hydrophilic solid supports. These structures exhibited an ultra-narrow diameter distribution and smooth walls and provided a reliable platform for investigation of how the substrate curvature affects the lipid mobility. The diffusion coefficients of the lipids on these tubular nanostructures were measured in the range of $2-10 \mu \mathrm{m}^{2} \mathrm{~s}^{-1}$, values similar to the diffusion coefficients of lipids on planar glass substrate, and the diffusion coefficient value increased further if the nanowire was narrowed down to $50 \mathrm{~nm}$. This trend was predicted by the free space diffusion model, which states that lipid molecules move faster when a more highly curved surface induces additional free space between the lipid head groups. Lipid vesicles are of significant interest because the total volume of a lipid vesicle is much smaller than $10^{-12} 1$, a dimension that cannot be achieved with conventional microfluidic devices. Vogel et al. ${ }^{39}$ synthesized and assembled attoliter molecular vessels into ordered arrays on a glass substrate using the microcontact printing 

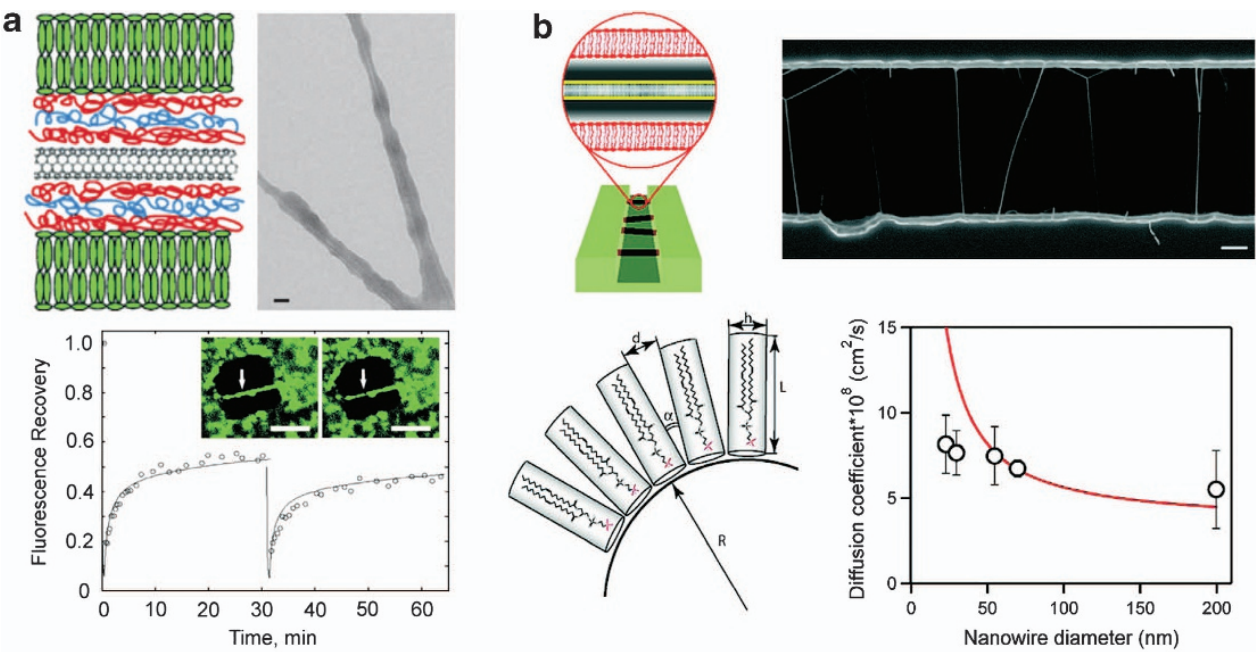

C
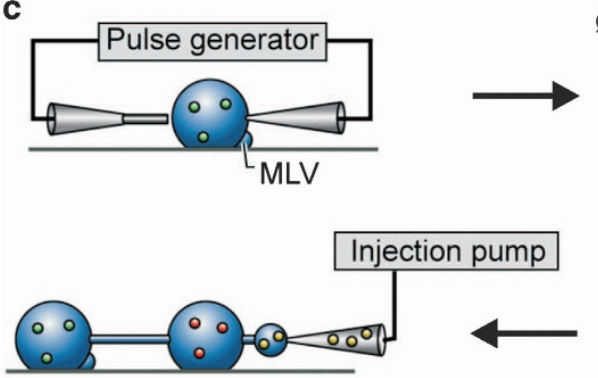

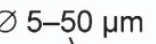

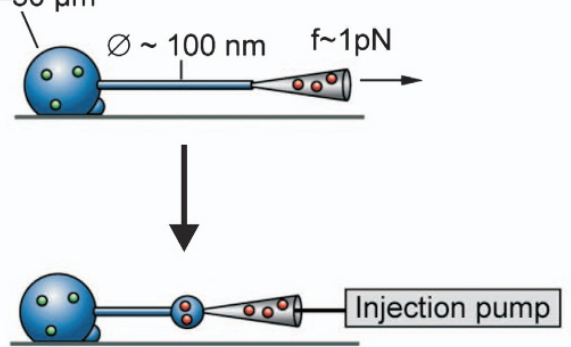

Figure 3 Lipid-one-dimensional (1D) nanomaterial hybrids. (a) Top: schematic illustration of polymer and lipid assembly on carbon nanotubes (CNTs). CNTs are coated with several alternating layers of oppositely charged polyelectrolytes, and a lipid bilayer is coated on the outermost surface. Transmission electron microscope (TEM) images of CNTs after coating with five alternating poly(diallyldimethylammonium chloride) (PDDA)/poly(sodium 4-styrene sulfonate) (PSS) layers. The scale bar is $20 \mathrm{~nm}$. Bottom: experimental (dot) and model (line) fluorescence recovery curves during two fluorescence recovery after photobleaching (FRAP) cycles on the same spot on lipid-coated nanotubes. The scale bar is $5 \mu \mathrm{m} .{ }^{37}$ (b) Top: schematic illustration of silicon-coated CNTs modified with lipid bilayers. SEM image of silicon-coated CNTs suspended over a 5- $\mu \mathrm{m}$-wide channel. Bottom: measured lipid mobility was compared with the free space diffusion model. ${ }^{38}$ (c) Schematic drawings showing micropipette-assisted formation and internal functionalization of networks of vesicles and nanotubes. ${ }^{47}$

technique and specific ligand-receptor interactions. Lipid vesicles immobilized on a solid surface have been used as a nanoreactor that isolates an ultra-small solution volume with the reactants and products. ${ }^{40-44}$ The controlled mixing and triggering of molecular reactions within the self-enclosed volume of immobilized vesicles could be beneficial, but the realization of these concepts is highly challenging. The giant unilamellar vesicle 1D lipid nanotube network systems allow the transport of reactive materials between containers and the initiation and control of chemical reactions in ultra-small volumes. ${ }^{13,45,46}$ The penetration of a giant uni-lamellar vesicle into a multi-lamellar membrane reservoir with a small buffer-filled glass pipette and subsequent mechanical pulling produced a lipid tube that connected the injection glass tip with the original vesicle. ${ }^{47} \mathrm{~A}$ new vesicle was observed to appear at the end of the lipid nanotube via the slow injection of buffer (Figure 3c). The lipids required for the growth of new vesicles were supplied from the attached multi-lamellar vesicles via a tensiondriven mechanism. Using this method, geometrically complex 3D lipid nanotube-vesicle networks were successfully fabricated. ${ }^{48}$

\section{Nanopore-lipid membrane hybrids}

Proper control over both the SLB formation and the nanostructured mechanical support can extend the distance between the lower leaflet of the membrane and the substrate in a freely suspended bilayer configuration. This arrangement provides opportunities to incorporate transmembrane proteins into a large extracellular domain in an active form as well as the use of a lower aqueous compartment for measuring the translocation events through the membrane. One of the most intensively investigated solid supports for freely suspended lipid bilayers is a porous membrane with a pore diameter ranging from $\sim 10$ to $\sim 100 \mathrm{~nm}$ (for example, anodic aluminumoxide, ${ }^{49}$ polycarbonate membranes ${ }^{50}$ and photolithographically generated silicon membranes). ${ }^{51,52}$ Depending on the surface of the porous supports, the bilayer is formed via the rupture of large or giant unilamellar vesicles and painting of lipids dissolved in organic solvent (Figure 4a). ${ }^{53}$ Such configurations are similar to the black lipid membrane in which planar lipid bilayers are formed across Teflon apertures with hole diameters of $0.1-1 \mathrm{~mm}$. In contrast to the black lipid membrane, the substantially reduced pore size gives the resulting lipid bilayers long-term stability and compatibility with the automated and miniaturized electronic devices. The mechanical decoupling between neighboring holes via the chemically immobilized lower leaflet of the bilayer on the rim of a hole causes the individual rupturing of the suspended bilayer, providing a longer lifetime for the devices than the classical black lipid membrane, which loses its entire function in a single rupture event. ${ }^{54}$ As the need for the development of more sophisticated ionchannel-based biosensing and high-throughput drug screening systems increases, a number of efforts have been aimed at achieving a $\mathrm{G} \Omega$ seal between two aqueous compartments separated by lipid bilayers and improving their lifetimes to several days. The high 

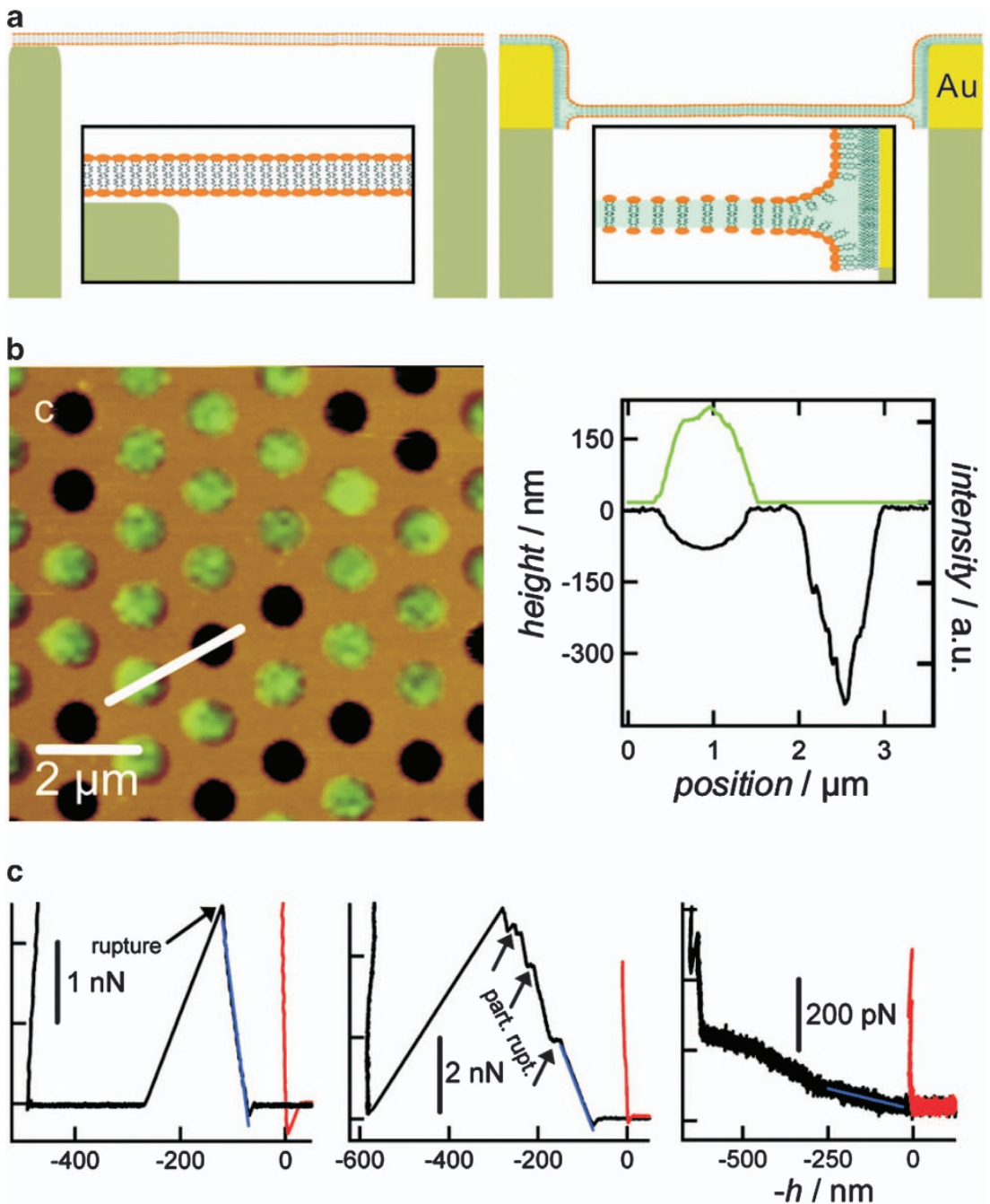

Figure 4 Lipid bilayer-nanopore hybrids. (a) Left: schematic illustration of solvent-free supported lipid bilayer (SLB) formed by spreading giant liposomes on a hydrophilic porous silicon substrate. Right: pore-spanning hybrid lipid bilayer coated through painting of lipids in organic solvent on octanethiol (OT)modified gold layers. ${ }^{53}$ (b) Left: correlation of atomic force microscopy (AFM) topography and fluorescence images of DPhPC/BodipyPC (99:1) on goldcoated porous silicon substrates with a pore radius of $400 \mathrm{~nm}$. The gold surface was modified with OT to form the hybrid lipid bilayers. Right: height and fluorescence profiles of membrane-covered and uncovered pores (white line in the left image). ${ }^{53}$ (c) Force indentation curves. The following legend indicates phospholipid/surface functionalization/pore radius/preparation method and the corresponding apparent spring constant $k_{\text {app }}$ (blue line). Curves, taken on the rim, are shown in red. Left: DPhPC/OT/400/n-decane, $k_{\text {app }}=40 \mathrm{mN} \mathrm{m}^{-1}$. Middle: DPhPC/OT/225/n-decane, $k_{\text {app }}=52 \mathrm{mN} \mathrm{m}^{-1}$. Right: DPhPC/silicon/250/ solvent-free SLB/, $k_{\text {app }}=230 \mu \mathrm{N} \mathrm{m}^{-1} .{ }^{53}$

membrane resistance required for a single ion channel measurement was attained by the formation of a lipid bilayer on a gold-coated porous alumina substrate with a surface was functionalized with 1,2dipalmitoyl-sn-glycero-3-phosphothioethanol. ${ }^{49}$ Although anodic aluminumoxide-SLBs showed good performance in terms of electrical insulation and mechanical stability, integration of these structures with a microfluidic system and control of the pore size and arrangement over a wide range are challenging tasks. In addition, the large aspect ratio of the anodic aluminumoxide suppresses the free diffusion of ions that are selectively transported by the ion channels, giving rise to an ionic concentration gradient and hampering free molecular access to the underside of the bilayer. A lithographically generated nanopore array in a silicon-based inorganic thin film has been suggested as an alternative platform to overcome these intrinsic limitations of anodic aluminumoxide supports. ${ }^{51,52,55}$ In an attempt to maximize the stability of a free-standing lipid bilayer, the effect of such chemical and structural parameters as the pore diameter of silicon and silicon nitride nanopore supports was investigated with atomic force microscopy ${ }^{52,53,56}$ (Figure $4 \mathrm{~b}$ ) and impedance spectroscopy. ${ }^{55}$ The results show that, in general, the bilayers suspended on the smaller pore arrays are more stable over time, and the aging stability of the lipid membranes is subject to the bilayer chemistry. Phosphatidyl ethanolamine bilayers are more stable than phosphatidyl choline bilayers because the cylindrical phosphatidyl ethanolamine lipids form a stable planar bilayer on the porous substrate, whereas the phosphatidyl choline lipids are energetically favored to exist in a curved form, such as a liposome. The degree of saturation of the lipid chains is also an important parameter in determining the mechanical properties of the bilayers. The mechanical behaviors of pore-spanning lipid bilayers were scrutinized by local indentation experiments with an atomic force microscopy tip (Figure 4c). ${ }^{53}$ The responses of the membranes were fully elastic, but the origins of these linear responses were varied depending on the preparation method and lipid composition. 


\section{LIPID-NANOSTRUCTURE HYBRIDS IN SENSING APPLICATIONS}

The noncovalent self-assembly of a lipid bilayer on the surface of a nanostructure enables the convergence between a highly sophisticated man-made platform and a nature-synthesized functional system. Membrane-associated molecular recognition events and ionic transport by membrane-incorporated ion channels take place in proximity to the surface of a cell membrane. This arrangement promotes the use of surface-sensitive working principles to detect the molecular binding event-induced change in a local environment close to the membrane. The LSPR, which is the coherent oscillations of conductive electrons on a metal surface, is sensitive to the change in the refractive index of a region within approximately $10 \mathrm{~nm}$ of the metal surface. ${ }^{57}$ When metallic nanostructures are intimately coupled to phospholipid membranes (typically approximately $5 \mathrm{~nm}$ in thickness), a change in the LSPR can probe the alteration in a target-bindinginduced refractive index change at the membrane-liquid interface. In a typical example, the binding of streptavidins to biotinylated lipidmembrane-coated gold nanorods was detected by monitoring the SPR spectra of nanoparticles with a imaging spectrometer coupled with a dark-field microscope (Figure 5a). ${ }^{20}$ Lipid membrane spreading and subsequent deposition of a full monolayer of streptavidins on the nanorods result in average plasmonic red-shifts of $3.6 \mathrm{~nm}$ and an additional $2.5 \mathrm{~nm}$, respectively. In a similar manner, Groves et al. ${ }^{58}$ reported target protein binding to glass-supported membranes in which hydrophobic alkanethiol self-assembled monolayer-modified silver nanocubes were embedded. This nanocube-membrane hybrid structure exhibited a calculated detection limit of $4.5 \mathrm{ng} \mathrm{cm}^{-2}$ and enables the dynamic kinetic analysis of target binding to cognate receptors in a label-free manner. These researchers further developed a solution-phase sensor for lipid-protein binding using SLB-assembled silver nanocubes with an ultra-thin silica shell. ${ }^{59}$ This label-free method is based on the LSPR of nanocubes, which enables the measurement of protein binding to a membrane surface on a standard laboratory spectroscopy setup. The protein binding to a membrane substrate induces a measurable change in the reflective index near the nanocube surface. The LSPR shifts were measured at varying surface protein densities, and a correlation was observed between the LSPR shift and the protein mass density, with a detection limit of $\sim 0.19 \mathrm{ng} \mathrm{cm}^{-2}$.

In other approaches, optically thin $\mathrm{Au}$ films perforated by randomly ordered nanoholes exhibited extinction peaks in the visible to near-infrared spectral range, which is closely associated with electronic polarization similar to the LSPR in metal nanoparticles. ${ }^{60}$ As the LSPR field from the dipolar resonance of a small cylindrical void is strongly confined to the holes, this optical configuration was well suited for sensing bio-recognition events that occur inside the metal holes. ${ }^{61}$ An orthogonal surface modification strategy that spatially confines the LSPR fields and receptors into the same small volume allows for the detection of various biomolecules in a zeptomole regime. ${ }^{62}$

Chemical fluorophores have been predominantly used as detection labels because they are commercially available, easy to incorporate into a lipid system and are observable on a conventional optical platform. Recently, plasmonic nanoparticles were tethered to lipid bilayers, and these particles showed good particle mobility on a lipid platform. These plasmonic labels have been considered to be an alternative optical label for studying membrane dynamics and heterogeneity. Molecular interactions in cell membranes are frequently involved in the rearrangement of membrane components, which induces a change in lipid mobility. ${ }^{63,64}$ This concept was realized on a SLB model membrane with a well-known toxin and receptor pair, cholera toxin and $\mathrm{GM}_{1}$. The target-binding-induced change in the membrane fluidity was evaluated using dark-field microscopy-based single nanoparticle tracking of gold nanoparticles tethered to lipids to determine the concentration of the target molecules in solution (Figure 5b). ${ }^{65} \mathrm{Nam}$ et al. used bovine serum albumin-modified paucivalent $\mathrm{Au}$ nanoparticles that have less open
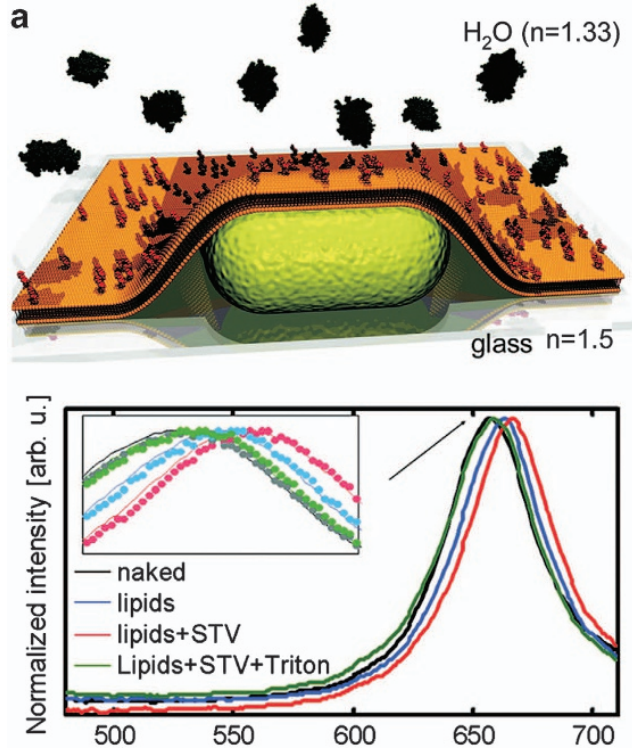

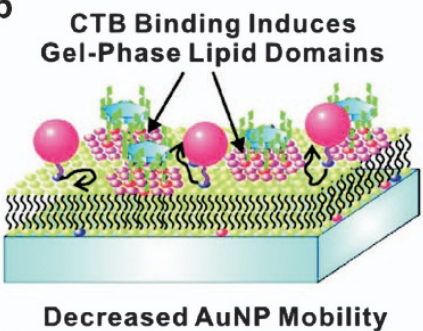

Due to Membrane-Bound CTBs

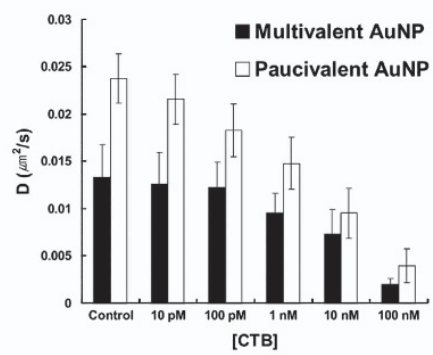

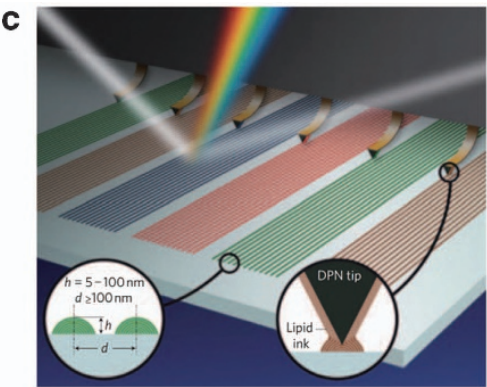

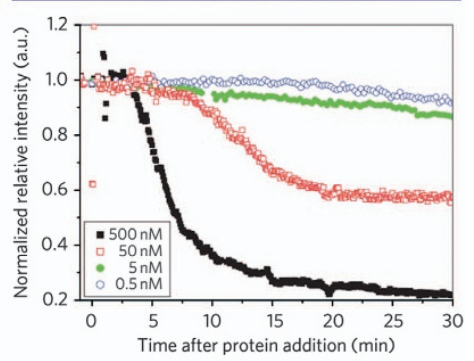

Figure 5 Lipid-nanostructure hybrid-based optical biosensors. (a) Top: schematic illustration of a gold nanorod (yellow) coated with a biotin(red)-incorporated lipid membrane (orange). Strepavidin is shown in green. Bottom: scattering spectra from single gold nanoparticles under various coating conditions. ${ }^{20}$ (b) Top: schematic illustration of two-dimensional diffusion of lipid-tethered gold nanoparticles exhibiting cholera toxin subunit B (CTB)-induced gel-fluid coexistence regime. Bottom: paucivalent gold nanoparticles more clearly differentiate CTB concentration-dependent change in diffusion coefficient than multivalent nanoparticle probes. ${ }^{65}$ (c) Top: schematic illustration of dip-pen nanolithography (DPN)-generated lipid gratings. Bottom: monitoring grating intensity allows labelfree detection of protein binding to receptor-modified lipid nanostructures. ${ }^{66}$ 

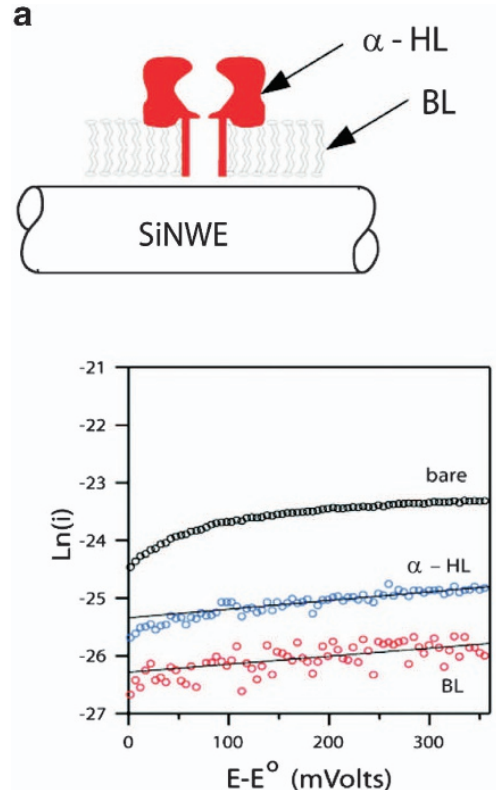
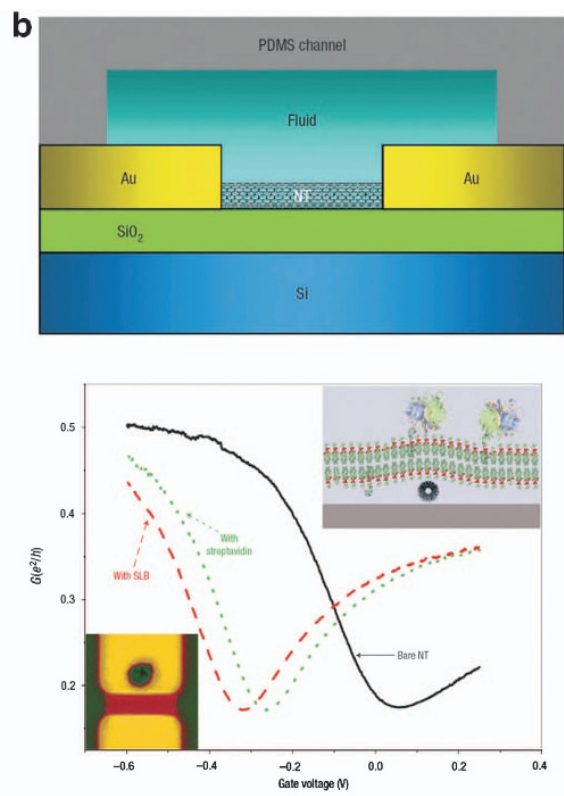
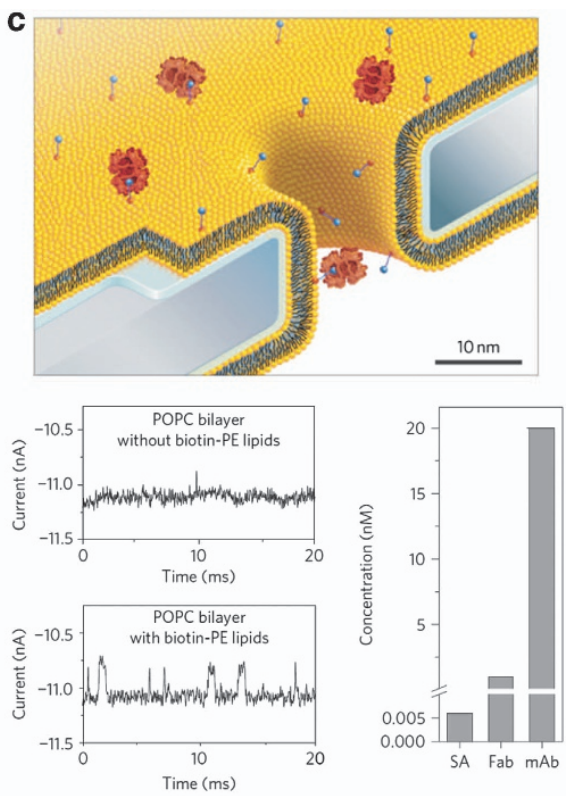

Figure 6 Lipid-nanostructure hybrid-based electrical biosensors. (a) Top: schematic illustration of an alpha-hemolysin pore-incorporated lipid bilayer-coated silicon nanowire. Bottom: electrochemical behaviors of a bare, lipid bilayer-coated nanowire and an alpha-hemolysin-containing lipid bilayer-coated single silicon nanowire. ${ }^{67}$ (b) Top: schematic illustration of the side view of SWNT FET integrated in a polydimethylsiloxane (PDMS) microfluidic channel. Bottom: coating of biotin-incorporated supported lipid bilayer (SLB) and accumulation of lipid-bound streptavidins (SAs) on a single-wall nanotube (SWNT) modulate the electrolyte-dependent conductance of a small-bandgap nanotube field-effect transistor (NT, FET). ${ }^{70}$ (c) Top: schematic illustration of translocation of biotin-SA complexes through the wall of a lipid bilayer-coated nanopore. Bottom left: current-versus-time traces show that the biotin-modified lipid bilayer dramatically increases translocation event frequency and slows down the translocation speed. Bottom right: minimum bulk concentrations required to observe at least 30-100 translocation events per second for SA, polyclonal anti-biotin Fab fragments (Fab) and monoclonal anti-biotin immunoglobulin G (lgG) antibodies. ${ }^{74}$

surface for lipid conjugation than multivalent bare gold nanoparticles to optimize the detection sensitivity to $\sim 100 \mathrm{pm}$. Unlike chemical fluorophores, these metal probes do not blink or photobleach and are stable for real-time and long-term observation purposes. These constructs should be useful in practical applications that require high sensitivity, a wide dynamic range and long-term and real-time monitoring of the cell-SLB interface.

Nanostructured lipid membranes allow for label-free optical detection of lipid-protein interactions in solution (Figure $5 \mathrm{c}$ ) ${ }^{66}$ In this system, biologically functional lipid multilayers were patterned with DPN on an optical waveguide surface to form optical grating structures that feature alternating lines with a periodicity comparable to the wavelength of visible light. On protein binding to receptors in a lipid grating, the structure is transformed by surface-tension-changeinduced de-wetting and protein intercalation as a function of the target concentration and grating height. This structural change affects the diffraction efficiency for protein concentrations as low as $500 \mathrm{pm}$.

One-dimensional nanostructures such as nanowires and nanotubes can be interfaced with cellular elements such as lipid bilayers and have been widely used as electrical biosensing platforms. For example, heavily doped silicon nanowires were coated with lipid bilayers that prevented the transport of redox-active species to the nanowire electrode surface, and these nanowire-lipid hybrid structures offer an effective and versatile bioelectrochemistry platform for detecting the insertion of ion channels (Figure 6a). ${ }^{67} \mathrm{~A}$ bacterium exotoxin $\alpha$-hemolysin-incorporated lipid bilayer on $\mathrm{Si}$ nanowires allows $\mathrm{Fe}(\mathrm{CN})_{6}{ }^{4-}$ ions to reach an electrode and results in an increase in the Faradic current. Selective ion transportation through gramicidin $\mathrm{A}$ and alamethicin pores reconstituted in lipid bilayers on Si nanowires were designed as a chemical and applied voltage-triggered electronic signal transduction model. ${ }^{68}$ Active ion transport was monitored by adopting additional biological components such as a protein ion pump and $\mathrm{Na}^{+} / \mathrm{K}^{+}$-ATPase on the surface of a CNT transistor. ${ }^{69}$ Instead of generating an ionic gradient across the membrane, the molecular binding to the SLB directly affects the electrical behavior of the underlying CNT field-effect transistor (FET) via surface charge modulation. One notable example is the detection of protein binding to cognate receptors in the SLB on a CNT FET (Figure $6 \mathrm{~b}$ ). ${ }^{70}$ In this system, the proteins on the membrane surface were accumulated near the single-walled CNTs via flow. The lipid molecules in the SLB freely move over the nanotube, whereas the protein on the membrane surface cannot move over the nanotube; therefore, the nanotube acted as a barrier for proteins. When streptavidin was added to interact with a biotinylated SLB and was driven near the CNT, the threshold voltage of the CNT FET changed to a more positive value. Graphene can be also coupled to a lipid membrane to construct membrane-graphene hybrid transistors. ${ }^{71}$ The antimicrobial peptide-induced membrane thinning brings the proximal membrane leaflets to within the Debye length of a $10-\mathrm{mm} \mathrm{NaF}$ solution, which gives rise to screening of the impurity potential from the coated lipid membrane and changes the electrical conductivity.

Functional ion channels were incorporated into a membrane and were supported by nanopores (spanning a few hundred nanometers) that hamper ionic transport across the membrane. This hybrid configuration can be used to study ligand-gated ion channels over the long term. A single bio-recognition event between an ion channellinked receptor and a specific ligand causes a number of ions to pass through the ion channel. Such biomimetic amplified electrical signal transduction allows for the electrochemical detection of ligand-gated single ion channel activity. ${ }^{49,72}$

Recently, nanopores of $<10$-nm have been used to detect individual biomolecules in a label-free and high-throughput manner. ${ }^{73}$ 

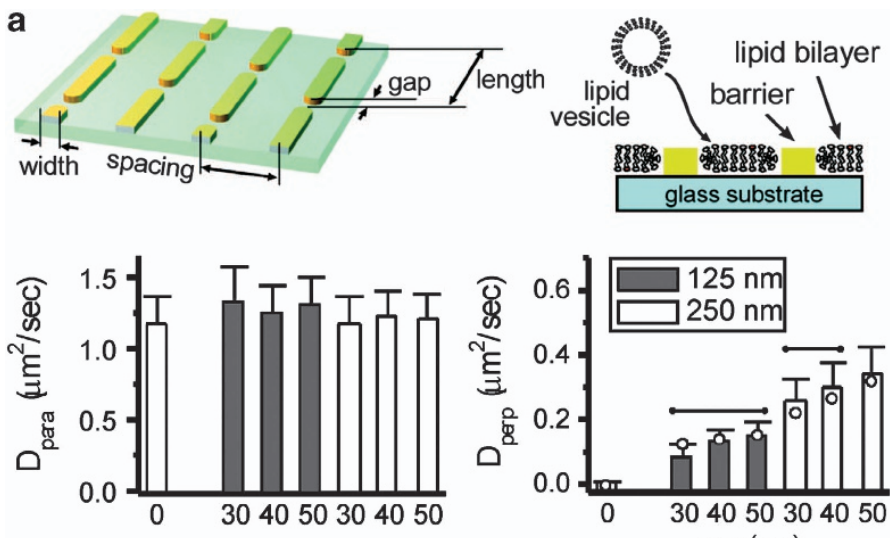

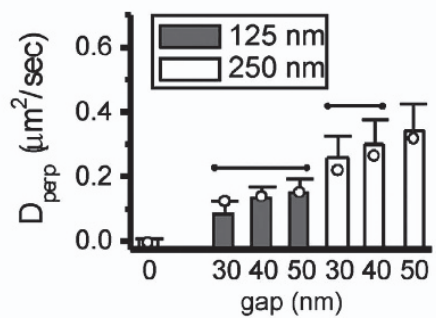

b
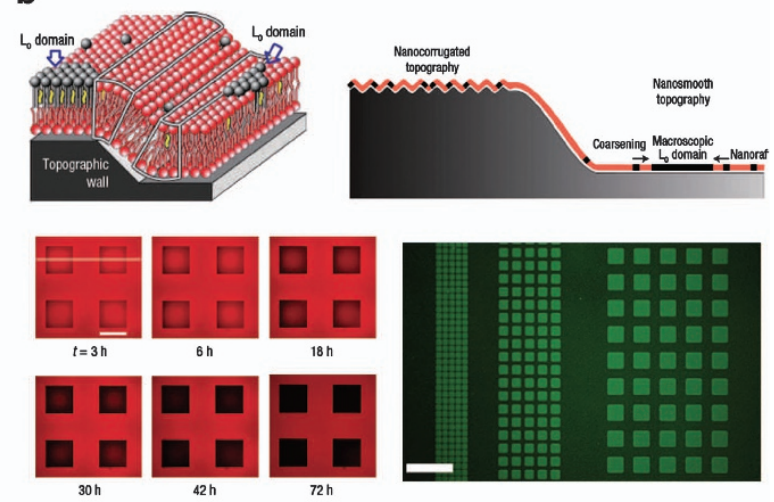

Figure 7 Lipid-nanostructure hybrids for lipid membrane modulation. (a) Top: schematics of a substrate with intermittent nanogaps (left) and cross-sectional view of a supported lipid bilayer (SLB) formed on a nanopatterned substrate. Bottom: diffusion coefficients of lipids in a perpendicular direction to metallic nanogap patterns (right) were significantly modulated compared with those in a parallel direction (left). ${ }^{77}$ (b) Top: SLB on a topographic wall creating elastic energy barriers at both side ends (white lines). Bottom left: time evolution of gel phase domain formation in an arrayed nanosmooth region. The epifluorescence signal comes from Texas-red 1,2-dihexadecanoyl-sn-glycero-3-phosphoethanolamine (DHPE). The scale bar is $200 \mu \mathrm{m}$. Bottom right: an epifluorescence microscope image of gel phase microdomain arrays reacted with Alexa 488 -CTB (cholera toxin subunit B). The scale bar is $200 \mu \mathrm{m} .{ }^{82}$

Recordings of the resistive current pulses during the translocation of single molecules, so-called Coulter counting, offers detailed information on the size, composition and conformation of biomolecules. For widespread use of nanopores in practical applications, however, there are many issues that must be addressed with respect to the precise fabrication of the nanopores at the sub-nanometer scale, the translocation time control and the analyte-specific translocation. In another example, a membrane-nanopore hybrid platform inspired by the olfactory sensilla of insect antennae was shown to provide a good solution when the nanopore surface was coated with a receptorincorporated fluid lipid bilayer (Figure 6c). ${ }^{74}$ The mobile receptors pre-concentrated the target proteins on the nanopore surface by confining the $3 \mathrm{D}$ diffusion of proteins to $2 \mathrm{D}$ diffusion. This process facilitates more frequent translocation events while slowing the translocation of target proteins, thus introducing chemical specificity and avoiding clogging.

\section{LIPID-NANOSTRUCTURE HYBRIDS FOR MODULATION OF LIPID MEMBRANE, NATIVE CELL MEMBRANE-MIMICKING AND NANO-BIO INTERFACING}

In native cell membranes, cytoskeletons and cytoskeleton-associated proteins form the semi-permeable barriers that selectively accept and exclude membrane constituents to build up the membrane domains that perform specific cellular functions. ${ }^{75}$ This process gives rise to the considerable modulation of molecular diffusion in cell membranes. Typically, diffusion is much more reduced by hop diffusion in which the short and long periods of diffusions reflect free and hindered motions, respectively. The introduction of intermittent nanogaps in diffusion barriers enables molecules governed by the hop diffusion mode to be found in native cell membranes.

Single molecule tracking of fluorescently labeled lipids on an $\mathrm{Ag}$ nanogap-modified substrate verified the hop diffusion mode with a certain escape probability. ${ }^{76} \mathrm{~A}$ similar approach to mimicking an anomalous diffusion of cell membrane has been reported. In that study, glass substrates were patterned with $50-\mathrm{nm}$ wide $\mathrm{Cr}$ lines with $125-\mathrm{nm}$ or 250-nm spacing and sub-50-nm gaps interspersed throughout the Cr barriers (Figure $7 \mathrm{a}$ ). ${ }^{77}$ In fluorescence recovery after photobleaching experiments, it was demonstrated that the longrange diffusion of the lipids on these substrates was significantly reduced, whereas the short-range diffusion was preserved. Furthermore, the cholera toxin subunit $\mathrm{B} / \mathrm{GM}_{1}$ complexes were less likely to cross the nanoscale barriers than the individual lipid molecules. This semi-permeable property is closely associated with the fundamental mechanism of enhancing the biological activity of a living cell membrane by increasing the local concentration of functional proteins. Although the diffusion barrier-patterned SLB enables precise control of the lateral diffusion mode in a manner similar to that of a native cell membrane, the static and complete separation between closure and opening sites with a discrete lipid bilayer is different from the native cell membrane structure, which features a continuous lipid membrane with active trafficking of a wide range of membrane components. The plasma membrane has the ability to bend and curve in 3D. ${ }^{78}$ Lipids, membrane-binding proteins and cytoskeletal proteins are involved in the control of membrane curvature, but membrane curvature also affects their structures and properties. Curvature in the membrane affects the basic principles of organizing membrane components into chemically specific functional assemblies. ${ }^{79}$ Vesicles, intercellular signaling junctions and filopodia adopt highly bent membrane structures. Such local radii of membrane curvatures at a nanometer scale abound in nature. ${ }^{80}$ Nanotopography can provide an SLB with a well-defined curvature that affects the lipid chain packing and phase separation in a manner similar to that of native cell membranes. In cell membranes, micrometer-sized rafts are formed through the coarsening process of the dynamic nanoscale assemblies of raft-forming components triggered by external signals or membrane trafficking. ${ }^{81}$ The coarsening of a $<10-\mathrm{nm}$ nanoraft into a macroscopic liquid-ordered domain was recapitulated in an arrayed format (Figure $7 \mathrm{~b}) .{ }^{82}$ In this work, the combination of nanocorrugated top surfaces and nanosmooth bottom surfaces separated by a topographic wall predefined the landscape of the energy barriers to suppress the coarsening process of the nanorafts on the top surface but allowed the promotion of selective formation of a micrometer-sized raft phase on the bottom of a well.

Cells interact with their environments through biochemical and mechanical cues. Typically, cell receptors in the membrane act as the initial contact points for incoming signals. These receptors bind to specific ligands, and these bindings induce signal transduction across 
a
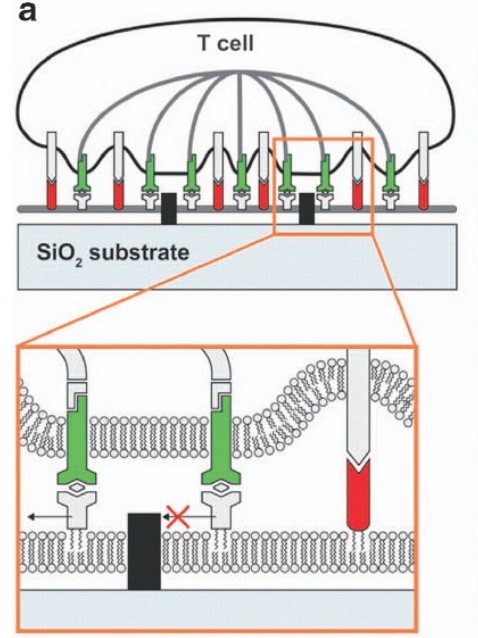

$\int \mathrm{TCR} \sum_{\pi}^{\circ} \mathrm{pMHC}$

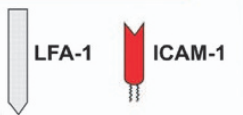

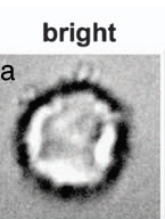
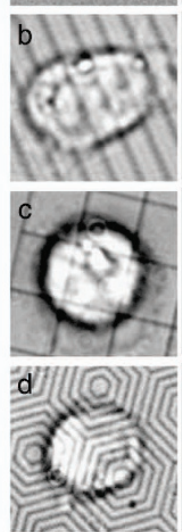

C

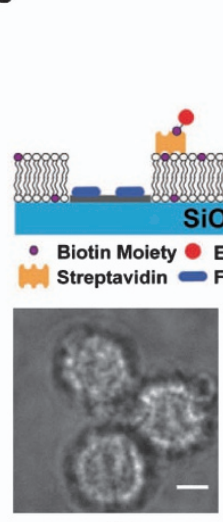

BF

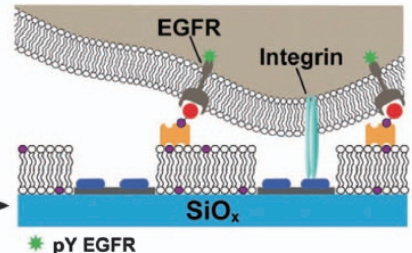

* PY EGFR

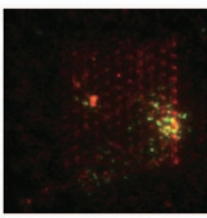

EGF/pY-EGFR

FN/pY-EGFR

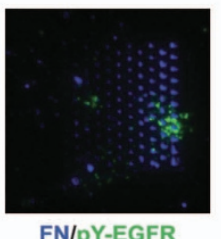

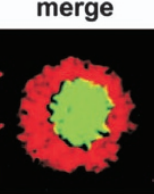
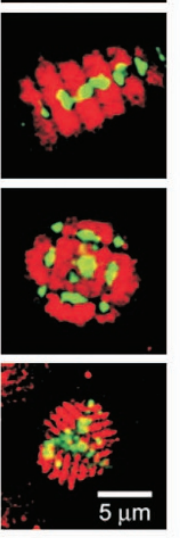

b
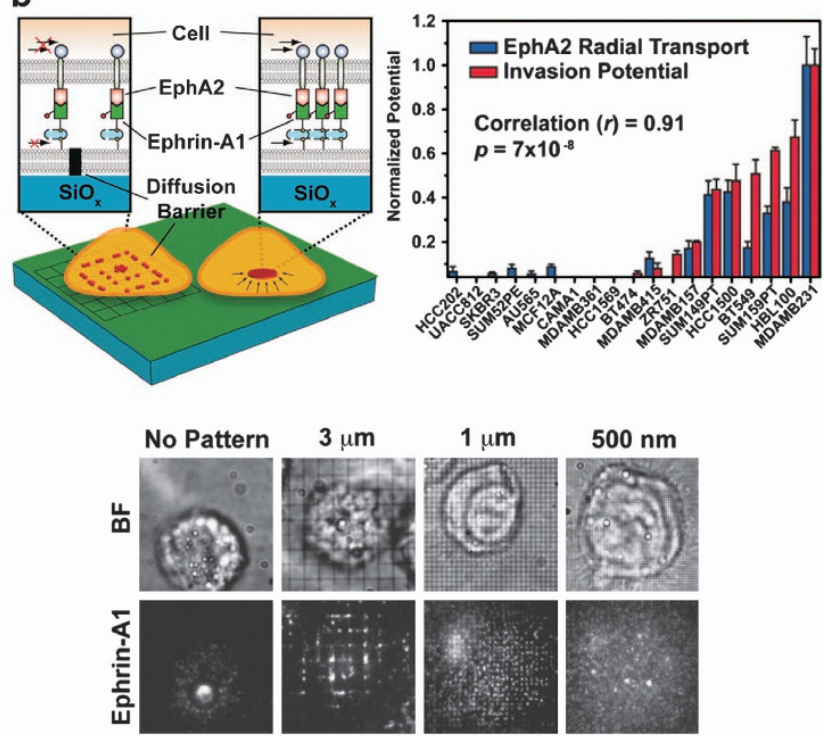

d
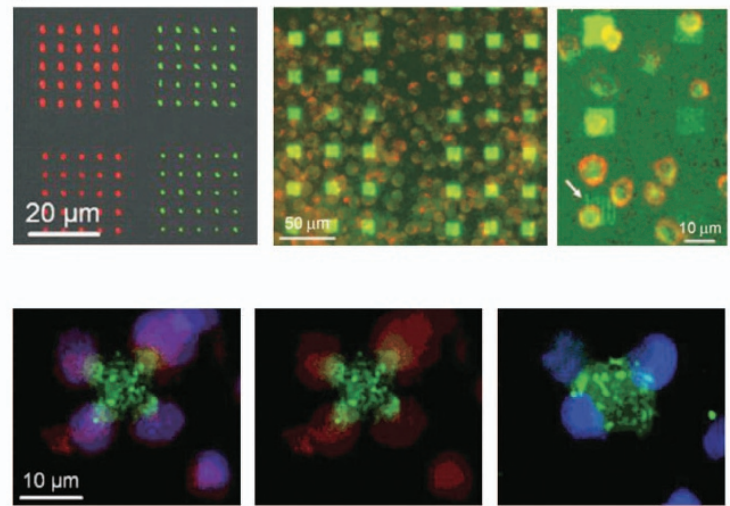

Figure 8 Interfacing synthetic lipid bilayers with live cells. (a) Left: schematic diagram of a live T-cell-supported membrane junction. Right: bright field and fluorescence images of the T cell and ligands. Synapse formation is altered by the geometrical constraints of the substrate. T-cell receptor (TCR; green) and intercellular adhesion molecule 1 (ICAM-1) (red) were labeled with fluorescent tags. ${ }^{63}$ (b) Left: experimental supported lipid bilayer (SLB) platform that triggers and manipulates the EphA2 receptors on the surface of living cells. Right: average ephrin-A1 ligand radial distribution functions for 26 cell lines are quantified and parameterized. The average radial distribution function was found to exhibit a strong correlation $\left(r=0.91, P=7 \times 10^{-8}\right)$ with invasion potentials that were determined with the modified Boyden chamber analysis. Bottom: images of fluorescently labeled ephrin-A1 ligands of EphA2-expressing mammary epithelial cells on a supported membrane. ${ }^{87}$ (c) Schematic illustration of the binary ligand system that presents immobilized FN-Cy3 and laterally mobile EGF-647. Representative brightfield, RICM, and fluorescence microscopy images of three cells that are engaged with a nanopatterned supported membrane. ${ }^{32}$ (d) The fluorescence image of phospholipid nanopatterns deposited on a glass surface. A three-channel image shows the T-cells adhering to the corners of lipid-protein dip-pen nanolithography (DPN) patterns and activated by anti-CD3/anti-CD28 (green $=$ lipid pattern, blue =the nucleus of cells by 4,6-diamidino-2-phenylindole (DAPI), red=CD69 by anti-CD69-phosphatidylethanolamine (PE) and anti-PE-tetramethylrhodamine-5(and-6)isothiocyanate (TRITC). ${ }^{30}$

the membrane to a cascade of chemical signaling reactions. ${ }^{83-85}$ The SLB has been used as a cell-surface-mimicking platform to stimulate and control immune cells, epithelia cells, cancer cells and neuronal cells via receptor binding and clustering. The immunological synapse formation of a $\mathrm{T}$ cell was reconstituted on the SLB surface that displays the peptide-major histocompatibility complex for mimicking an antigen-presenting cell. ${ }^{86}$ The physical constraints of receptorligand complexes imposed by patterned lipid bilayers provide important insights into cell-cell interactions. For example, T-cell receptor (TCR) microclusters were identified as the active signaling units, and other key factors such as actin were heavily recruited in the signaling process. ${ }^{63}$ In this study, glycosylphosphatidylinositol-linked peptide-major histocompatibility complex and intercellular adhesion molecule 1-modified SLB on a nanoscale Cr-patterned substrate were used as an artificial antigen-presenting cell surface (Figure 8a). The TCRs interacted with peptide-major histocompatibility complex and intercellular adhesion molecule 1 on the artificial antigen-presenting cell surface, a necessary process for antigen recognition by living $\mathrm{T}$ cells. The TCR-ligand complexes on the SLB were subject to the geometrical restriction on mobility imposed by the $\mathrm{Cr}$ lines. The effect of spatial constraints on synaptic signaling through TCR clustering using the Cr barrier-nanopatterned membrane support was demonstrated. In addition, the EphA2 signaling pathway of cancer cells was regulated spatially and mechanically by a Cr metal line nanofabricated substrate created via electron-beam lithography (Figure $8 \mathrm{~b}$ ). ${ }^{87}$ The EphA2, a receptor tyrosine kinase, is overexpressed in $\sim 40 \%$ of 
human breast cancer cells. EphA2 dimerization occurs through binding to a membrane-anchored ephrin-A1. This EphA2-ephrinA1 complex is known to have important roles in cancer development. After receptor-ligand engagement, the signaling cascade undergoes the recruitment of several signaling molecules. The spatial organizations of the receptor-ligand complexes of 26 mammary epithelial cell lines on a nanopatterned SLB surface were displayed and analyzed quantitatively. The results revealed that the difference in the spatial organization of the complexes is strongly correlated with the invasion potential of a cancer cell. In another study, the EphA2-ephrin-A1 juxtacrine signaling interaction was studied using a gold nanoparticlearrayed SLB. The MDA-MB-231 cells, a highly invasive human breast epithelial cancer cell line, were cultured on the nanoparticleembedded supported bilayer substrate. ${ }^{34}$ In this article, the coupling efficiency to the nanoparticles and the influence of a fixed nanoparticle array on the surrounding membrane integrity were characterized and studied in detail. Salaita et al. observed the organization of epidermal growth factor receptors (EGFRs) ${ }^{30}$ and adhesion receptors on a cell surface using a DPN-patterned SLB platform with fibronectin and epidermal growth factor (EGF). The EGFR-overexpressed breast cancer cells, HCC1143, were cultured on this surface to observe the rearrangement of cell surface receptors along with the nanopatterned SLB. The researchers observed the formation of EGF-EGFR clusters that translocated to the center of the cell surface junction, presumably through a retrograde flow mechanism and the motion restriction of EGFR in the cell membrane by poly(diallydimethylammonium chloride)-fibronectin adsorbates (Figure $8 \mathrm{c}$ ). In another example, DPN-generated protein-tethered lipid nanopatterns on a glass substrate were used to activate Jurkat T cells. ${ }^{32}$ The multilayered lipid patterns were stable under cell culture conditions, and the biomimetic function of the protein-bound lipid patterns was demonstrated by testing the ability of the nanopatterns to activate $\mathrm{T}$ cells. This group modified two different types of activating antibodies specific for the CD3 e chain of the TCRs (a-CD3e), the co-stimulatory receptor (a-CD28). Jurkat $\mathrm{T}$ cells were selectively adhered to the corners of square patterns, and the activation of $\mathrm{T}$ cells was confirmed by CD69 expression through fluorescence imaging (Figure 8d).

\section{CONCLUSIONS AND PERSPECTIVES}

We have introduced and reviewed various lipid-nanostructure hybrids with respect to fabrication strategies and the use of these platforms in the study of biological molecules and cells. The lipids in the SLB act not only as cell membrane elements but also as fluid molecules that can be dynamically and actively assembled and disassembled. Nanostructures are particularly interesting because they intrinsically possess nanoscale-based properties (that is, optical, electrical and magnetic properties) and sizes that match well with biological structures and many biological events, such as the clustering of biomolecules that occur on a nanometer scale. Furthermore, many different biologically functional nanometer-scale structures and features can simultaneously interface with a microscale cell, which offers opportunities for controlling and studying cells of interest on a manipulable platform. By interfacing these nanostructures with lipids, the main components of the cell membrane, new opportunities become possible for the study and analysis of many biologically important issues, such as cell-cell communications. Many examples have shown that these hybrid structures can successfully reproduce such biological events as the spatial organization of ligands and receptors, membrane curvature and phase segregation in a controllable manner. These advances usher in new findings in biology that could not be discovered using conventional platforms. However, for more practical use and realization of the full potential of these systems, we must now integrate these elements into functional devices, solve the lipid bilayer stability issues caused by the structural fragility of SLB in harsh conditions (such as dehydration and high salt concentration) and address nonspecific binding, especially for biosensing and cell-interfacing applications. ${ }^{88,89}$ Furthermore, incorporating functional proteins such as transmembrane proteins into a lipid layer in an in vivo-like configuration while sustaining their functionalities is highly difficult. Finally, current techniques for generating nanoscale cell membrane features are mostly based on the creation of single heterogeneous membrane structures. For the creation of more complex and nature-like structures, multiple spatial configurations of multiple cellular components should be generated and controlled as a function of biologically relevant size and time scale. ${ }^{90,91}$ The fabrication of analytical biosensor-integrated cell-interfacing nanostructures also provides the possibility of multi-functional hybrid platforms and allows for in situ study of cellular responses induced by various stimuli, such as nanoscale local exposure to signaling molecules, topography, and electrical and thermal signals. For real-time and long-term live cell study, it is also critical to develop new nanoprobes that can address the issues related to chemical fluorophores, including photobleaching and photoblinking.

\section{ACKNOWLEDGEMENTS}

J-M Nam was supported by a National Research Foundation (NRF) of Korea grant (no. 2011-0018198) and the Pioneer Research Center Program (no. 2012-0009565) through the NRF of Korea funded by the Ministry of Science, ICT \& Future Planning. We also acknowledge the financial support from the Industrial Strategic Technology Development Program (no. 10033183 and 10037397) funded by the Ministry of Trade, Industry \& Energy (MI, Korea).

1 Bangham, A. D., Standish, M. M. \& Watkins, J. C. Diffusion of univalent ions across lamellae of swollen phospholipids. J. Mol. Biol. 13, 238-252 (1965).

2 Tamm, L. K. \& McConnell, H. M. Supported phospholipid bilayers. Biophys. J. 47, 105-113 (1985).

3 Brian, A. A. \& McConnell, H. M. Allogeneic stimulation of cytotoxic T cells by supported planar membranes. Proc. Nat. Acad. Sci. 81, 6159-6163 (1984).

4 Chan, Y. H. M. \& Boxer, S. G. Model membrane systems and their applications. Curr. Opin. Chem. Biol. 11, 581-587 (2007).

5 Castellana, E. T. \& Cremer, P. S. Solid supported lipid bilayers: from biophysical studies to sensor design. Surf. Sci. Rep. 61, 429-444 (2006).

6 Tanaka, M. \& Sackmann, E. Polymer-supported membranes as models of the cell surface. Nature 437, 656-663 (2005)

7 Lee, Y. K. \& Nam, J. -M. Electrofluidic lipid membrane biosensor. Small 8, 832-837 (2012).

8 Nam, J. -M., Nair, P. M., Neve, R. M., Gray, J. W. \& Groves, J. T. A fluid membranebased soluble ligand-display system for live-cell assays. Chembiochem. 7, 436-440 (2006).

9 Liu, C. M., Monson, C. F., Yang, T. L., Pace, H. \& Cremer, P. S. Protein separation by electrophoretic-electroosmotic focusing on sported Ipid bilayers. Anal. Chem. 83, 7876-7880 (2011).

10 Henzie, J., Grünwald, M., Widmer-Cooper, A., Geissler, P. L. \& Yang, P. Self-assembly of uniform polyhedral silver nanocrystals into densest packings and exotic superlattices. Nat. Mater. 11, 131-137 (2012).

11 Nel, A. E., Madler, L., Velegol, D., Xia, T., Hoek, E. M. V., Somasundaran, P., Klaessig, F., Castranova, V. \& Thompson, M. Understanding biophysicochemical interactions at the nano-bio interface. Nat. Mater. 8, 543-557 (2009).

12 Lee, J. -H., Kim, G. -H. \& Nam, J. -M. Directional synthesis and assembly of bimetallic nanosnowmen with DNA. J. Am. Chem. Soc. 11, 5456-5459 (2012).

13 Jesorka, A. \& Orwar, O. Liposomes: technologies and analytical applications. Ann. Rev. Anal. Chem. 1, 801-832 (2008).

14 Bally, M., Bailey, K., Sugihara, K., Greshaber, D., Vörös, J. \& Städler, B. Liposome and lipid bilayer arrays towards biosensing applications. Small 6, 2481-2497 (2010).

15 Richter, R. P., Berat, R. \& Brisson, A. R. Formation of solid-supported lipid bilayers: an integrated view. Langmuir 22, 3497-3505 (2006).

16 Roiter, Y., Ornatska, M., Rammohan, A. R., Balakrishnan, J., Heine, D. R. \& Minko, S. Interaction of nanoparticles with lipid membrane. Nano Lett. 8, 941-944 (2008). 
17 Roiter, Y., Ornatska, M., Rammohan, A. R., Balakrishnan, J., Heine, D. R. \& Minko, S Interaction of lipid membrane with nanostructured surfaces. Langmuir 25, 62876299 (2009).

18 Mornet, S., Lambert, O., Duguet, E. \& Brisson, A. The Nano Lett.formation of supported lipid bilayers on silica nanoparticles revealed by cryoelectron microscopy. Nano Lett. 5, 281-285 (2005).

19 Pfeiffer, I., Seantier, B., Petronis, S., Sutherland, D., Kasemo, B. \& Zach, M. Influence of nanotopography on phospholipid bilayer formation on silicon dioxide. J. Phys. Chem B. 112, 5175-5181 (2008).

20 Baciu, C. L., Becker, J., Janshoff, A. \& Sonnichsen, C. Protein-membrane interaction probed by single plasmonic nanoparticles. Nano Lett. 8, 1724-1728 (2008).

21 Jonsson, M. P., Jonsson, P., Dahlin, A. B. \& Hook, F. Supported lipid bilayer formation and lipid-membrane-mediated biorecognition reactions studied with a new nanoplasmonic sensor template. Nano Lett. 7, 3462-3468 (2007).

22 Hovis, J. S. \& Boxer, S. G. Patterning barriers to lateral diffusion in supported lipid bilayer membranes by blotting and stamping. Langmuir 16, 894-897 (2000).

23 Groves, J. T., Ulman, N. \& Boxer, S. G. Micropatterning fluid lipid bilayers on solid supports. Science 275, 651-653 (1997)

24 Boxer, S. G. \& Groves, J. T. Micropattern formation in supported lipid membranes. Acc. Chem. Res. 35, 149-157 (2002).

25 Groves, J. T., Ulman, N. \& Boxer, S. G. Micropatterning fluid lipid bilayers on solid supports. Science 275, 651-653 (1997).

26 Kam, L. \& Boxer, S. G. Formation of supported lipid bilayer composition arrays by controlled mixing and surface capture. J. Am. Chem. Soc. 122, 12901-12902 (2000).

27 Nair, P. M., Salaita, K., Petit, R. S. \& Groves, J. T. Using patterned supported lipid membranes to investigate the role of receptor organization in intercellular signaling. Nat. Prot 6, 523-539 (2011)

28 Salaita, K., Wang, Y. H. \& Mirkin, C. A. Applications of dip-pen nanolithography. Nat. Nanotech. 2, 145-155 (2007)

29 Salaita, K., Wang, Y. H., Fragala, J., Vega, R. A., Liu, C. \& Mirkin, C. A. Massively parallel dip-pen nanolithography with 55000-pen two-dimensional arrays. Angew. Chem. Int. Ed. 45, 7220-7223 (2006).

30 Narui, Y. \& Salaita, K. S. Dip-pen nanolithography of optically transparent cationic polymers to manipulate spatial organization of proteolipid membranes. Chem. Sci $\mathbf{3}$, 794-799 (2012)

31 Lenhert, S., Sun, P., Wang, Y. H., Fuchs, H. \& Mirkin, C. A. Massively parallel dip-pen nanolithography of heterogeneous supported phospholipid multilayer patterns. Small $\mathbf{3}$, 71-75 (2007).

32 Sekula, S., Fuchs, J., Weg-Remers, S., Nagel, P., Schuppler, S., Fragala, J., Theilacker, N., Franzreb, M., Wingren, C., Ellmark, P., Borrebaeck, C. A., Mirkin, C. A., Fuchs, H. \& Lenhert, S. Multiplexed lipid dip-pen nanolithography on subcellular scales for the templating of functional proteins and cell culture. Small 4, 1785-1793 (2008).

33 Shi, J. J., Chen, J. X. \& Cremer, P. S. Sub-100 nm patterning of supported bilayers by nanoshaving lithography. J. Am. Chem. Soc. 130, 2718-2719 (2008).

34 Lohmuller, T., Triffo, S., O'Donoghue, G. P., Xu, Q., Coyle, M. P. \& Groves, J. T. Supported membranes embedded with fixed arrays of gold nanoparticles. Nano Lett. 11, 4912-4918 (2011).

35 Zheng, G. F., Patolsky, F., Cui, Y., Wang, W. U. \& Lieber, C. M. Multiplexed electrical detection of cancer markers with nanowire sensor arrays. Nat. Biotechnol. 23, 1294-1301 (2005)

36 Richard, C., Balavoine, F., Schultz, P., Ebbesen, T. W. \& Mioskowski, C. Supramolecular self-assembly of lipid derivatives on carbon nanotubes. Science $\mathbf{3 0 0 , 7 7 5 - 7 7 8}$ (2003)

37 Artyukhin, A. B., Shestakov, A., Harper, J., Bakajin, O., Stroeve, P. \& Noy, A. Functiona one-dimensional lipid bilayers on carbon nanotube templates. J. Am. Chem. Soc. 127 7538-7542 (2005)

38 Huang, S. C. J., Artyukhin, A. B., Martinez, J. A., Sirbuly, D. J., Wang, Y., Ju, J. W., Stroeve, P. \& Noy, A. Formation, stability, and mobility of one-dimensional lipid Bilayers on polysilicon nanowires. Nano Lett. 7, 3355-3359 (2007)

39 Stamou, D., Duschl, C., Delamarche, E. \& Vogel, H. Self-assembled microarrays of attoliter molecular vessels. Angew. Chem. Int. Ed. 42, 5580-5583 (2003).

40 Bolinger, P. -Y., Stamou, D. \& Vogel, H. Intergrated nanoreactor systems: triggering the release and mixing of compounds inside single vesicles. J. Am. Chem. Soc. 126, 8594-8595 (2004)

41 Pick, H., Schmid, E. L., Tairi, A. P., Ilegems, E., Hovius, R. \& Vogel, H. Investigating cellular signaling reactions in single attoliter vesicles. J. Am. Chem. Soc. 127 2908-2912 (2005)

42 Bolinger., P. -Y., Stamou, D. \& Vogel, H. An integrated self-assembled nanofluidic system for controlled biological chemistries. Angew. Chem. Int. Ed. 47, 5544-5549 (2008)

43 Cisse, I., Okumus, B., Joo, C. \& Ha, T. Single-molecule chemistry and biology special feature: fueling protein-DNA interactions inside porous nanocontainers. Proc. Nat. Acad. Sci 104, 12646-12650 (2007).

44 Cristensen, S. M., Bolinger, P. -Y., Hatzakis, N. S., Mortensen, M. W. \& Stamou, D. Mixing subattolitre volumes in a quantitative and highly parallel manner with soft matter nanofluidics. Nat. Nanotech. 7, 51-55 (2011).

45 Karlsson, R., Karlsson, A., Ewing, A., Dommersnes, P., Joanny, J. F., Jesorka, A. \& Orwar, 0. Chemical analysis in nanoscale surfactant networks. Anal. Chem. 78, 5960-5968 (2006)

46 Karlsson, M., Davidson, M., Karlsson, R., Karlsson, A., Bergenholtz, J., Zoran, K., Jesorka, A., Lobovkina, T., Hurtig, J., Voinova, M. \& Orwar, O. Biomemetic nanoscale reactors and networks. Annu. Rev. Phys. Chem. 55, 613-649 (2004).
47 Jesorka, A., Stepanyants., N., Zhang, H. J., Ortmen, B., Hakonen, B. \& Orwar, O Generation of phospholipid vesicle-nanotube networks and transport of molecules therein. Nat. Prot 6, 791-805 (2011).

48 Hurtig, J., Karlsson, M. \& Orwar, O. Topographic SU 8 substrates for immobilization of three-dimensional nanotube-vesicle networks. Langmuir 20, 5637-5641 (2004).

49 Schmitt, E. K., Vrouenraets, M. \& Steinem, C. Channel activity of OmpF monitored in nano-BLMs. Biophys. J. 91, 2163-2171 (2006).

50 Favero, G., Campanella, L., Cavallo, S., D’Annibale, A., Perrella, M., Mattei, E. \& Ferri, T. Glutamate receptor incorporated in a mixed hybrid bilayer lipid membrane array, as a sensing element of a biosensor working under flowing conditions. J. Am. Chem. Soc. 127, 8103-8111 (2005).

51 Romer, W., Lam, Y. H., Fischer, D., Watts, A., Fischer, W. B., Goring, P., Wehrspohn, R. B., Gosele, U. \& Steinem, C. Channel activity of a viral transmembrane peptide in micro-BLMs: Vpu1-32 from HIV-1. J. Am. Chem. Soc. 126, 16267-16274 (2004).

52 Simon, A., Girard-Egrot, A., Sauter, F., Pudda, C., D'Hahan, N. P., Blum, L., Chatelain F. \& Fuchs, A. Formation and stability of a suspended biomimetic lipid bilayer on silicon submicrometer-sized pores. J. Colloid Interface Sci. 308, 337-343 (2007).

53 Mey, I., Stephan, M., Schmitt, E. K., Müller, M. M., Ben Amar, M., Steinem, C. \& Janshoff, A. Local membrane mechanics of pore-spanning bilayers. J. Am. Chem. Soc. 131, 7031-7039 (2009)

54 Weiskopf, D., Schmitt, E. K., Kluhr, M. H., Dertinger, S. K. \& Steinem, C. Micro-BLMs on highly ordered porous silicon substrates: rupture process and lateral mobility. Langmuir 23, 9134-9139 (2007).

55 Han., X., Studer, A., Sehr, H., Geissbuhler, I., Di Berardino, M., Winkler, F. \& Tiefenauer, L. Nanopore arrays for stable and functional free-standing lipid bilayers. Adv. Mater. 19, 4466-4470 (2007).

56 Steltenkamp, S., Muller, M. M., Deserno, M., Hennesthal, C., Steinem, C. \& Janshoff, A. Mechanical properties of pore-spanning lipid bilayers probed by atomic force microscopy. J. Biophysical 91, 217-226 (2006).

57 Willets, K. A. \& Van Duyne, R. P. Localized surface plasmon resonance spectroscopy and sensing. Annu. Rev. Physical Chem. 58, 267-297 (2007)

58 Galush, W. J., Shelby, S. A., Mulvihill, M. J., Tao, A., Yang, P. \& Groves, J. T. A nanocube plasmonic sensor for molecular binding on membrane surfaces. Nano Lett. 9, 2077-2082 (2009).

59 Wu, H. -J., Henzie, J., Lin, W. -C., Rhodes, C., Li, Z., Sartorel, E., Thorner, J., Yang, P. \& Groves, J. T. Membrane-protein binding measured with solution-phase plasmonic nanocube sensors. Nat. Methods 9, 1189-1191 (2012).

60 Prikulis, J., Hanarp, P., Olofsson, L., Sutherland, D. \& Kall, M. Optical spectroscopy of nanometric holes in thin gold films. Nano Lett. 4, 1003-1007 (2004).

61 Dahlin, B., Jonsson, P. \& Hook, F. Specific self-assembly of single lipid vesicles in nanoplasmonic apertures in gold. Adv. Mater 20, 1436-1442 (2008).

62 Dahlin, A., Zach, M., Rindzevicius, T., Kall, M., Sutherland, D. S. \& Hook, F. Localized surface plasmon resonance sensing of lipid-membrane-mediated biorecognition events. J. Am. Chem. Soc. 127, 5043-5048 (2005).

63 Mossman, K. D., Campi, G., Groves, J. T. \& Dustin, M. L. Altered TCR signaling from geometrically repatterned immunological synapses. Science 310, 1191-1193 (2005).

64 Hayden, C. C. Hwang, J. S., Abate, E. A. Kent, M. S. \& Sasaki, D. Y. Directed formation of lipid membrane microdomains as high affinity sites for His-tagged proteins. J. Am. Chem. Soc. 131, 8728-8729 (2009).

65 Yang, Y. -H. \& Nam, J. -M. Single nanoparticle tracking-based detection of membrane receptor-ligand Interactions. Anal. Chem. 81, 2564-2568 (2009).

66 Lenhert, S., Brinkmann, F., Laus, T., Walheim, S., Vannahme, C., Klinkhammer, S., Xu M., Sekula, S., Mappes, T., Schimmel, T. \& Fuchs, H. Lipid multilayer gratings. Nat. Nanotech 5, 275-279 (2010).

67 Martinez, J. A., Misra, N., Wang, Y., Stroeve, P., Grigoropoulos, C. P. \& Noy, A. Highly efficient biocompatible single silicon nanowire electrodes with functional biologica pore channels. Nano Lett. 9, 1121-1126 (2009).

68 Misra, N., Martinez, J. A., Huang, S. -C. J., Wang, Y., Stroeve, P., Grigoropoulos, C. P. \& Noy, A. Bioelectronic silicon nanowire devices using functional membrane proteins. Proc. Nat. Acad. Sci 106, 13780-13784 (2009).

69 Huang, S. C. J., Artyukhin, A. B., Misra, N., Martinez, J. A., Stroeve, P. A., Grigoropoulos, C. P., Ju, J. -W. W. \& Noy, A. Carbon nanotube transistor controlled by a biological ion pump gate. Nano Lett. 10, 1812-1816 (2010).

70 Zhou, X., Moran-Mirabal, J. M., Craighead, H. G. \& McEuene, P. L. Supported lipid bilayer//carbon nanotube hybrids. Nat. Nanotech 2, 185-190 (2007).

71 Ang, P. K., Jaiswal, M., Lim, C. H. Y. X., Wang, Y., Sankaran, J., Li, A., Lim, C. T. Wohland, T., Barbaros, O. \& Loh, K. P. A bioelectronic platform using a graphene-lipid bilayer interface. Acs Nano 4, 7387-7394 (2010).

72 Reimhult, E. \& Kumar, K. Membrane biosensor platforms using nano- and microporous supports. Trends in Biotech 26, 82-89 (2008).

73 Dekker, C. Solid-state nanopores. Nat. Nanotech. 2, 209-215 (2007).

74 Yusko, E. C., Johnson, J. M., Majd, S., Prangkio, P., Rollings, R. C., Li, J., Yang, J. \& Mayer, M. Controlling protein translocation through nanopores with bio-inspired fluid walls. Nat. Nanotech 6, 253-260 (2011).

75 Verhey, K. J. \& Hammond, J. W. Traffic control: regulation of kinesin motors. Nat. Rev. Mol. Cell Biolo 10, 765-777 (2009).

76 Takimoto, B., Nabika, H. \& Murakoshi, K. Single molecular observation of hop diffusion in a lipid bilayer at metallic nanogates. J. Physic. Chem. C 113, 3127-3132 (2009).

77 Tsai, J., Sun, E., Gao, Y., Hone, J. C. \& Kam, L. C. Non-brownian diffusion of membrane molecules in nanopatterned supported lipid bilayers. Nano Lett. 8 425-430 (2008)

78 McMahon, H. T. \& Gallop, J. L. Membrane curvature and mechanisms of dynamic cell membrane remodelling. Nature 438, 590-596 (2005). 
79 Parthasarathy, R. \& Groves, J. T. Curvature and spatial organization in biological membranes. Soft Matter 3, 24-33 (2007).

80 Antonny, B. Mechanisms of membrane curvature sensing. Annu. Rev. Biochem. 80, 101-123 (2011).

81 Hancock, J. F. Lipid rafts: contentious only from simplistic standpoints. Nat. Rev. Mol. Cell Biol. 7, 456-462 (2006).

82 Yoon, T. -Y., Jeong, C., Lee, S. -W., Kim, J. H., Choi, M. C., Kim, S. -J., Kim, M. W. \& Lee, S. -D. Topographic control of lipid-raft reconstitution in model membranes. Nat. Mater. 5, 281-285 (2006)

83 Saito, T. \& Yokosuka, T. Immunological synapse and microclusters: the site for recognition and activation of T cells. Curr. Opin. Immunol. 18, 305-313 (2006).

$84 \mathrm{He}$, L., Fong, J., von Zastrow, M. \& Whistler, J. L. Regulation of opioid receptor trafficking and morphine tolerance by receptor oligomerization. Cell 108, 271-282 (2002).

85 Grakoui, A., Bromley, S. K., Sumen, C., Davis, M. M., Shaw, A. S., Allen, P. M. \& Dustin, M. L. The immunological synapse: a molecular machine controlling $T$ cell activation. Science 285, 221-227 (1999).

86 Grakoui, A., Bromley, S. K., Sumen, C., Davis, M. M., Shaw, A. S., Allen, P. M. \& Dustin, M. L. The immunological synapse: a molecular machine controlling $T$ cell activation. Science 285, 221-227 (1999).
87 Salaita, K., Nair, P. M., Petit, R. S., Neve, R. M., Das, D., Gray, J. W. \& Groves, J. T. Restriction of receptor movement alters cellular response: physical force sensing by EphA2. Science 327, 1380-1385 (2010).

88 Persson, F., Fritzsche, J., Mir, K. U., Modesti, M., Westerlund, F. \& Tegenfeldt, J. O. Lipid-based passivation in nanofluidics. Nano Lett. 12, 2260-2265 (2012).

89 Huang, Y., Palkar, P. V., Li, L. J., Zhang, H. \& Chen, P. Integrating carbon nanotubes and lipid bilayer for biosensing. Biosens. Bioelectron. 25, 1834-1837 (2010).

90 Kashimura, Y., Furukawa, K. \& Torimitsu, K. Electrostatic control of lipid bilayer self-spreading using a nanogap gate on a solid support. J. Am. Chem. Soc. 133, 6118-6121 (2011).

91 Jackson, B. L., Nye, J. A. \& Groves, J. T. Electrical manipulation of supported lipid membranes by embedded electrodes. Langmuir 24, 6189-6193 (2008).

(c) (i) () () $९$ This work is licensed under a Creative Commons
Attribution-NonCommercial-NoDerivs 3.0 Unported License. To view a copy of this license, visit http://creativecommons. org/licenses/by-nc-nd/3.0/

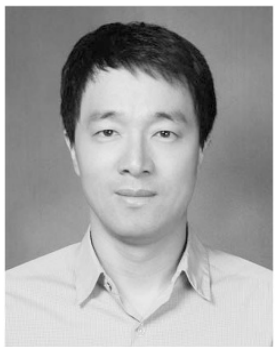

Professor Jwa-Min Nam received his BS and MS degrees in chemistry from Hanyang University, Korea. He obtained his PhD in chemistry from Northwestern University and worked as a postdoctoral fellow at the University of California, Berkeley. He joined the Department of Chemistry, Seoul National University, in 2006 as an Assistant Professor and was promoted to Associate Professor in 2010. Professor Nam received the Presidential Young Scientist Award from the President of the Republic of Korea and the Korean Academy of Science and Technology (2012); the Young Inorganic Chemist Award from the Korean Chemical Society (2012); the Outstanding Basic Science Research Achievement Award from the Ministry of Education, Science and Technology of the Republic of Korea (2010); the Victor K LaMer Award from the American Chemical Society (2006); and the Collegiate Inventors Award from the National Inventors Hall of Fame, USA (2004). Professor Nam has served as a consultant for Nanosphere, Inc. and as a scientific advisory board member for Medifron DBT. His major interests include the synthesis and optics of plasmonic nanostructures, nanocarriers for bio-imaging and delivery, nanoprobe-tethered lipid bilayers, cell-nanostructure interfaces, graphene chemistry and biosensors.

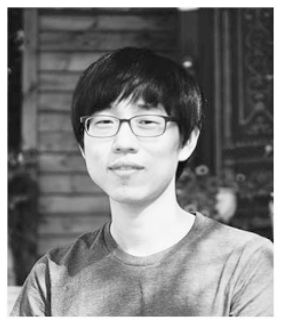

Young Kwang Lee received his BS and MS degrees in chemistry at Kookmin University, Korea. He obtained his PhD in Chemistry from Seoul National University. His current research subjects focus on the development of actively controllable supported lipid bilayer platforms for biosensing and plasmonics applications.

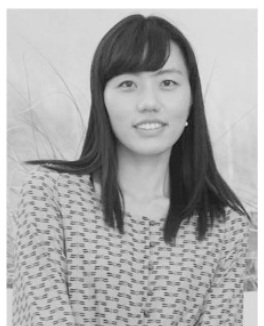

Dr Hyojin Lee received her PhD degree from the Department of Chemistry from Seoul National University, under the supervision of Professor Jwa-Min Nam in 2012. Her main research focus has been the development of nanostructure fabrication and manufacturing of a bio-mimetic system for biological application. She is currently working as a postdoctoral researcher at Seoul National University. 